\title{
Adaptive Traffic Parameter Prediction: Effect of Number of States and Transferability of Models
}

\author{
Gurcan Comert ${ }^{\mathrm{a}, *}$, Anton Bezuglov ${ }^{\mathrm{b}}$, Mecit Cetin $^{\mathrm{c}}$ \\ ${ }^{a}$ Physics and Engineering Department, Benedict College, 1600 Harden St., Columbia, SC 29204 USA \\ ${ }^{b}$ Math and Computer Science Department, Benedict College, 1600 Harden St., Columbia, SC 29204 USA \\ ${ }^{c}$ Civil and Environmental Engineering Department, Old Dominion University, 135 Kaufman Hall, Norfolk, VA 23529 USA
}

\begin{abstract}
Traffic parameters can show shifts due to factors such as weather, accidents, and driving characteristics. This study develops a model for predicting traffic speeds under these abrupt changes within regime switching framework. The proposed approach utilizes Hidden Markov, Expectation Maximization, Recursive Least Squares Filtering, and ARIMA methods for an adaptive forecasting method. The method is compared with naive and mean updating linear and nonlinear time series models. The model is fitted and tested extensively using 1993 I-880 loop data from California and January 2014 INRIX data from Virginia. Analysis for number of states, impact of number of states on forecasting, prediction scope, and transferability of the model to different locations are investigated. A 5-state model is found to be providing best results. Developed model is tested for 1-step to 45-step forecasts. The accuracy of predictions are improved until 15-step over nonadaptive and mean adaptive models. Except 1-step predictions, the model is found to be transferable to different locations. Even if the developed model is not retrained on different datasets, it is able to provide better or close results with nonadaptive and adaptive models that are retrained on the corresponding dataset.
\end{abstract}

Keywords: Hidden Markov model, speed prediction, time series ARIMA forecasting, change point models, recursive least squares filtering

\section{Problem definition}

Intelligent Transportation Systems (ITS) applications such as Advanced Traveler Information Systems (ATIS), Freight Advanced Traveler Information Systems (FRATIS), real-time route guidance, and emergency response systems planning seek to improve the efficiency of transportation networks (i.e., safety, environment, economic competitiveness, via enhancing decision making capabilities of the system users). Accurate and reliable traffic parameter (e.g., speed, travel time) prediction can be used in real-time routing to find the fastest way to a destination and to avoid congestion for on-time delivery

\footnotetext{
${ }^{*}$ Corresponding author
} 
which can lead to efficient transportation networks where people and goods are mobilized at minimum travel time, fuel consumption, and emissions. These systems, however, demand for highly robust traffic parameter predictions. The accuracy and reliability become more important for large scale network-level applications. In real life, traffic may exhibit unexpected or expected changes due to various factors such as driver behaviors, incidents, inclement weather, special events, and demand surges which adversely affect the performance of forecasting models. This paper presents an online adaptive traffic parameter prediction model based on regime switching and numerically tests if it can be transferred to different locations (transferability), be used for prediction scopes, and effectively handle nonlinearities in the process by monitoring and switching the candidate forecasting models (Clements et al. (2004)).

Regime switching concept simply consists of changing the parameters of a certain model fit. This framework originally presented by Goldfeld and Quandt (1973); Cosslett and Lee (1985); Hamilton (1989). Based on thorough review studies, regime-switching models can be divided into two categories of threshold and Markov-switching models (Potter (1999); Kuan (2002); Piger (2009)). The primary differences are number of changes and how they evolve overtime. Threshold models treat the shifts as triggered by the level of observed variables (usually the same lagged response variable) through smooth transitioning threshold parameters. These models, originally introduced by Tong (1990), are used to model long lasting structural breaks. Main disadvantages can be listed as fitting procedure can be challenging with possible local optimums in parameter optimization and unable to adapt to different change patterns (Kuan (2002)). More towards modeling correlated data with distinct patterns during different time periods (repetitive temporary shifts), Markov-switching models are introduced within econometrics. In Markov switching framework, possibly multiple regime shifts are treated as stochastic process which are abrupt and evolve according to a Markov chain-an unobserved discrete random variable. Thus, changes are determined through statistical inference rather than an observed specific data. In traffic flow, data generation processes (DGP) that contain stable segments with unknown break points indicating the instances when the dynamic behavior switches from one regime to another due to discrete shifts are common. They can be mainly caused by accidents, roadwork, and sudden demand surges. Certainly, fitted model parameters need to be updated in relation to these break points. However, updating parameters continuously may not be necessary while they are not changing statistically. Making unnecessary updates which entails online optimization problems, is not a computationally efficient strategy. As in regime switching models, it would be appropriate to devise a statistical test to monitor changes and update parameters conditional on the occurrence of changes. Calculating these updates and effectively detecting abrupt changes also require careful identification of observations belonging to different regimes (states).

In this research, the main objective is to treat the traffic data with unknown breaks and develop 
an adaptive prediction model for traffic parameters through incorporating machine learning algorithms as detection and adapting tools. The approach falls into above-mentioned Markov switching category. Technically, Hidden Markov model (HMM) is allocated to calculate the conditional probability of being in a certain traffic speed state given past observations. It provides the detection of possible shifts in the DGP and switch of forecasting models. Number of states (i.e., mixture distributions) are optimized using parts of the datasets. Time series autoregressive integrated moving average (ARIMA) models are fit to each speed condition and utilized as the main forecasting tool. Expectation Maximization (EM) is employed to update mean levels (intercepts) of ARIMA models. It is able to quickly detects a change from a normal traffic speed level alleviating the problem identified in Piger (2009). Recursive Least Squares (RLS) algorithm is used to filter the parameters of ARIMA models in order to replicate the recalibration of forecasting model parameters. The impact of different geographical locations in terms of freeways in two States (California and Virginia) and multiple detector sets on a freeway is tested. This is referred as transferability of the methods (HMM, ARIMA, EM, and RLS parameters) to other locations without retraining. A through recalibration can be done for changed factors such as varying facility types, speed limits, number of lanes, or anything that can permanently alter the state-speed representation and/or the transitional probabilities of the HMM. Practically, the recalibration or updating can be carried out given the low HMM training time (i.e., less than 20 seconds PC with 8GB of memory, Pentium I3 Quad-Core CPU). However, such recalibration of HMM or RLS parameters are not dealt within this paper. Major assumption for the proposed model is an underlying Normal distribution with constant variance for the data generation process within each state.

\subsection{Literature review}

In the literature, many researchers have proposed a number of parametric and nonparametric methods for short-term traffic prediction. Parametric models mainly include time series, their variations, and Kalman Filter $(\mathrm{KF})$ models. Nonparametric methods consist of nonparametric regression and neural networks (NN). For detailed reviews of the various short-term forecasting models and their critical aspects, the reader is referred to the work by Smith and Demetsky (1997); Smith et al. (2002); Vlahogianni et al. (2004); Vlahogianni and Karlaftis (2011); Vlahogianni et al. (2014). In sum, these studies report that the non-parametric techniques have superior performance to simple time series models. However, it is acknowledged that higher computational power and volume of data are demanded. Major advantages of the parametric approach are well-developed theory and ability to interpret the parameters. Generally, parametric prediction methods first train a model using historical data to estimate parameters and then test it on different data. Main assumption in this parametric modeling approach is unchanged process characteristics (e.g., mean and variance) which may affect the prediction accuracy. Since traffic is subject 
to occasional abrupt disturbances (e.g., incidents and weather) or congestion level (see Sun and Zhou (2005); Kamarianakis et al. (2010)) that can potentially change the underlying dynamics of the data generation process. Therefore, robust forecasting models are needed that incorporate the changes when they occur.

Hybrid methods have been used by researchers to obtain adaptive models. Cetin and Comert (2007) combine an ARIMA model with Expectation Maximization (EM) and Cumulative Summation (CUSUM) algorithms to update the intercept. Qi and Ishak (2014); Noroozi and Hellinga (2014) apply Hidden Markov models (HMM) for traffic speed predictions. Elhenawy and Rakha (2014) identify the congestion from mixtures of skewed speed distributions. Adaptive fuzzy logic and Bayesian credit assignment algorithms in Dimitriou et al. (2008) and Min and Wynter (2011) are applied to select the best NN flow predictor at a given period. Min and Wynter (2011) present an extended time series model that includes spatial and temporal correlations for full time range network level flow forecast in urban networks. Tan et al. (2009) offer an aggregate traffic flow prediction model that combines Exponential Smoothing, Moving Average, and ARIMA (Autoregressive Integrated Moving Average) based on a NN and calls for the best model for a given period. Kindzerske and Ni (2007) use nonparametric regression with NN for backward propagating shockwave forecasting. In addition to the mean level forecasting, there have been new studies on the volatility to generate confidence bounds (Kamarianakis et al. (2005); Guo and Williams (2010); Guo et al. (2014b); Zhang et al. (2014)). Karlaftis and Vlahogianni (2009) develop a fractional integrated process to uncover long term structure of time series and apply the model on traffic volume prediction. The study fits an Autoregressive Fractionally Integrated Moving Average (ARFIMA) with Fractionally Integrated Volatility Process (FIGARCH) model and states that the model better represents the transportation time series compared to the classical ARIMA although very large time series needed for these models. Dimitriou et al. (2008) give an adaptive fuzzy rule-based meta-optimized model for flow forecasting at urban networks. The model is provided with offline, online, univariate, and multivariate options. Model parameters are optimized by a genetic algorithm and the results with a classical ARIMA, KF, offline univariate, multivariate, online univariate, and multivariate models are compared. The best accuracy is obtained from online multivariate model with highest $R^{2}$ value. A detailed study by Kim and Mahmassani (2014) recently uses finite mixture models to classify network level vehicle-to-vehicle and day-to-day variability of travel times.

Regime switching, mixtures, or applications of change point models in other fields are vastly common especially in finance, economics, engineering, and sciences (Clements and Hendry (1996); Hamilton (2008, 2010); Clements and Hendry (2011); Castle et al. (2011); Aiolfi et al. (2011); Gu et al. (2013); Ruggieri (2013); Carpenter et al. (2014); Keshavarz and Huang (2014)). In transportation, two state mean adapting 
model with EM is developed in (Cetin and Comert (2007) and Comert and Bezuglov (2013)) combined a four state HMM, EM, and ARIMA (i.e., Online Change Point Based (OCPB) model) and compared against mean adapting and simple time series models. It is found that the developed model better represents average traffic speed behavior. Similarly, different forecasting models of ARIMA, KF, and back propagation NN via Bayesian model are combined in (Wang et al. (2014)) and Guo et al. (2014a) give a three-stage framework with data smoothing, machine learning methods, and an error feedback structure. Liu et al. (2014) combine empirical mode decomposition and ARIMA models. Differently in this paper, effect of number of states on HMM and speed predictions is investigated; impact of a filtering algorithm for parameters is presented; transferability of the model to other locations without retraining is discussed; capability of long term predictions are given; and extended number of forecasting models are compared with the developed method. Some of which are discussed as new challenges to the researchers in short-term forecasting (Vlahogianni et al. (2014)).

The rest of the paper is organized as follows. Section 2 introduces components of the proposed Online Change Point Based Model with RLS (OCPBr). In subsections 2.1, 2.2, 2.3, and 2.4, HMM, ARIMA, Expectation Maximization, and RLS methods and their roles in OCPBr are explained. Section 3 includes the detailed numerical experiments for training and testing of the method. The section describes the data used, presents the optimum number states discussion, and provides comparisons of prediction performances against naive, linear adaptive, seasonal, and nonlinear time series methods. In addition, detailed results are provided for transferability and long-term prediction performances of the proposed model. Finally, section 4 summarizes findings and addresses possible future research directions.

\section{Methodology}

A simple flow diagram is given for the proposed method in Fig. 1. It is essentially an hybrid adaptive traffic parameter prediction model that consists of four major components. A change point algorithm (Hidden Markov model) that is used to detect shifts and to switch forecasting models. The intuitive idea is to calculate the conditional distribution of a certain state given recent observations as well as learned parameters from historical data. After training the Hidden Markov model for optimum number of states, conditional probabilities based on underlying distribution assumption (i.e., Normal Distribution in this study) are calculated. Since, ARIMA models are theoretically well explored, simple, and parametric methods, for main forecasting tool, time series ARIMA models are utilized for each state identified in the DGP. Then, parameters of fitted time series models are updated by EM and RLS algorithms. Mean level (intercept) update is carried out with EM. Although HMM is able to provide conditional probabilities online, state mean and variances are only obtained after training. Therefore for an online level update, 
it is observed that EM is able to provide better mean level updates compared to HMM's weighted state parameters with transition probabilities. This is expected as EM with low number of data inputs (i.e., 10) can easily converge to desired number of mixtures with updated parameters at each time step. Recursive least squares is used for adaptive filtering of ARIMA model parameters except the intercept. Since states are determined in HMM, any major differences in terms of parameters would be addressed in this stage. ARIMA models are fitted state-by-state, however, minor parameter changes occurring within a state especially of long runs, a filtering algorithm can be used. So for continuous and longer prediction implementations, filtering can certainly be beneficial. Following subsections thoroughly explain these methods and their roles within the proposed model.

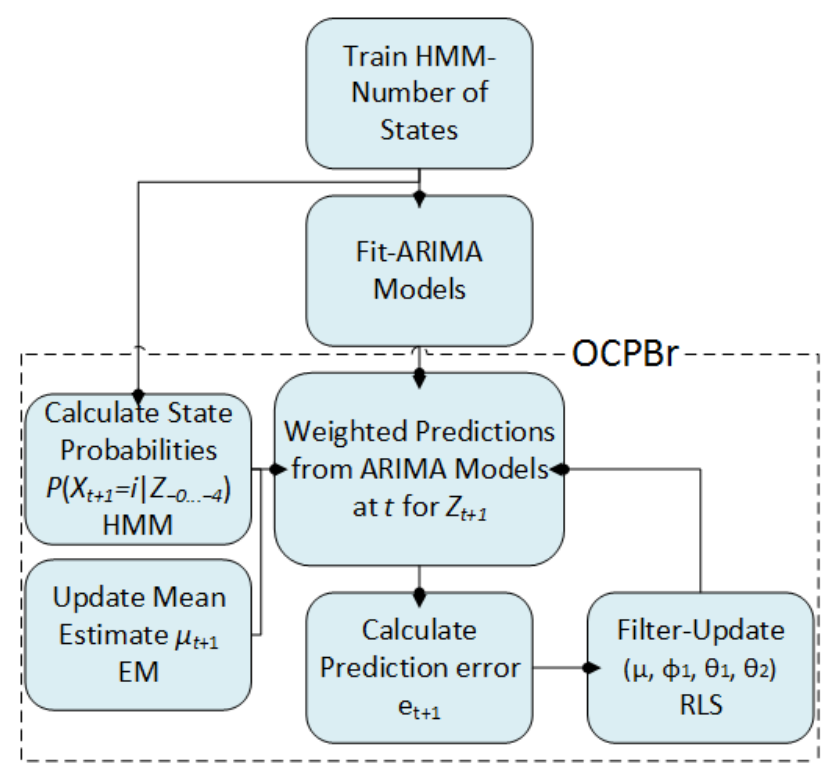

Fig. 1. Flow of the OCPBr model

\subsection{Hidden Markov model}

Hidden Markov model (HMM) is a machine learning method that is appropriate to the problem of change point detection (Hamilton (2008); Qi (2010); Hamilton (2010)). The model consists of a hidden state variable $X_{t}$ and an observation variable $Z_{t}$ at time $t$. The state of the model at time $t$ depends only on the previous state of the model at immediate past $X_{t-1}$ through distribution $P\left(X_{t} \mid X_{t-1}\right)$. The observation variable $Z_{t}$ depends on the current state $X_{t}$. Three probability distributions constitute the behavior of the model: $P\left(X_{0}\right)$ is the initial state, $P\left(X_{t} \mid X_{t-1}\right)$ denotes how the state changes, and $P\left(Z_{t} \mid X_{t}\right)$ represents how the state influences the observation. The observable variable $Z_{t}$ represents aggregated one-minute average traffic speed at time $t . Z_{t}$ is assumed to be normally distributed for each state $X_{t}$ : $P\left(Z_{t} \mid X_{t}=i\right)=N\left(\mu_{i}, \sigma_{i}\right)$. Under these assumptions, two conditions need to be identified to apply HMM to change point detection (Rabiner (1989)): 
1. Given a model $\lambda$ and a sequence of observations $Z_{1 . . t}$, maximize the likelihood of the assigned states $X_{1 . . t}$

2. Given a set of observations $Z_{1 . . t}^{i}$, find an HMM with $\lambda^{*}$ that will maximize the likelihood of the set $L(Z ; \lambda)=P\left(Z_{1 . . t} \mid \lambda\right)$, i.e. training HMM.

The usual procedure for solving the problems above is to calculate forward $\alpha_{t}(i)$ and backward $\beta_{t}(i)$ variables using Forward-Backward algorithm (Baum and Eagon (1967)). These variables represent the probabilities to finish (forward) or to start (backward) of an HMM at state $i$ at time $t$ given the observations $Z_{1 . . T}$ :

$$
\alpha_{t}(i)=P\left(Z_{1 . . t}, X_{t}=i \mid \lambda\right) \quad \beta_{t}(i)=P\left(Z_{t+1 . . T}, X_{t}=i \mid \lambda\right)
$$

Forward-Backward algorithm suggests an iterative procedure to calculate the variables:

$$
\begin{gathered}
\alpha_{1}(i)=\pi_{i} \cdot b_{i}\left(Z_{1}\right) \quad \alpha_{t+1}(i)=\left(\sum_{j=1}^{N} \alpha_{t}(j) \cdot a_{i j}\right) \cdot b_{i}\left(Z_{t+1}\right) \\
\beta_{T}(i)=1 \quad \beta_{t}(i)=\sum_{j=1}^{N} a_{i j} \cdot b_{j}\left(Z_{t+1}\right) \cdot \beta_{t+1}(j)
\end{gathered}
$$

Where $\pi_{i}$ is the probability of the model to start at state $i: \pi_{i}=P\left(X_{1}=i\right)$. In this algorithm, $b_{i}$ is the probability of observing $Z_{1}$ at state $i: b_{i}=P\left(Z_{t}=Z_{t} \mid X_{t}=i\right)$. Finally, $a_{i j}$ represents the switching behavior of the HMM: $a_{i j}=P\left(X_{t+1}=i \mid X_{t}=j\right)$. To solve the first problem and find the state sequence with the highest likelihood, variable $\gamma_{t}(i)$ (a normalized product of $\alpha_{t}(i)$ and $\beta_{t}(i)$ ) is introduced:

$$
\gamma_{t}(i)=\frac{\alpha_{t}(i) \cdot \beta_{t}(i)}{\sum_{j=1}^{N} \alpha_{t}(j) \cdot \beta_{t}(j)}
$$

As a result, the state sequence with the highest likelihood at each time $t$ is: $X_{t}=\arg \max _{1 \leq i \leq N} \gamma_{t}(i)$. In addition, the Forward-Backward algorithm is used to train HMM (finding the best fitting HMM) using $\xi_{t}(i, j)=P\left(X_{t}=i, X_{t+1}=j, Z \mid \lambda\right)$ which is calculated by

$$
\xi_{t}(i, j)=\frac{\alpha_{t}(i) \cdot a_{i j} \cdot b_{j}\left(Z_{t+1}\right) \cdot \beta_{t+1}(j)}{\sum_{i=1}^{N} \sum_{j=1}^{N} \alpha_{t}(i) \cdot a_{i j} \cdot b_{j}\left(Z_{t+1}\right) \cdot \beta_{t+1}(j)}
$$

Both variables $\gamma_{t}(i)$ and $\xi_{t}(i, j)$ allow to calculate expected values for HMM parameters $\bar{\pi}_{i}=\gamma_{1}(i), \overline{a_{i j}}$, and $b_{j} \overline{(k)}$ given the observed data. It has been demonstrated (Baum et al. (1970)) that when these expected values are used as the new HMM parameters, its likelihood improves. If this re-estimation step is used iteratively (i.e., the Baum-Welch algorithm), then within several iterations the likelihood reaches a local maximum and the HMM is considered as trained. This procedure is in fact a special application of EM algorithm (Dempster et al. (1977)). The discussion of training and the impact of number of states on HMM's performance are presented in subsection 3.1.1. 


\subsection{Time series forecasting models}

Time series ARIMA models are commonly used forecasting tool in the literature (Brook and Evans (1972); Hamilton (2010)). These models handle time correlated forecasts by exploiting the autocorrelation structures in time series data. ARIMA models and their extensions have been applied to the traffic data before (Williams (2001)). The notation for an $\operatorname{ARIMA}(p, d, q)(P, D, Q)_{S}$ with seasonal component can be written as follows:

$$
\phi_{p}(B) \Phi_{P\left(B^{S}\right)}(1-B)^{d}\left(1-B^{S}\right)^{D} Z_{t}=\theta_{q}(B) \Theta_{Q(B)} a_{t}
$$

Where $B=$ backshift operator defined by $B^{j} Z_{t}=Z_{t-j}$,

$\phi_{p}(B)=\left(1-\phi_{1} B-\ldots-\phi_{p} B^{p}\right)=$ autoregressive $(\mathrm{AR})$ polynomial of order $p$,

$\Phi_{p}\left(B^{S}\right)=\left(1-\Phi_{1} B^{S}-\ldots-\Phi_{p} B^{P S}\right)=$ seasonal autoregressive(AR) polynomial of order $P$,

$\theta_{q}(B)=\left(1-\theta_{1} B-\ldots-\theta_{q} B^{q}\right)=$ moving average(MA) polynomial of order $q$,

$\Theta_{Q}(B)=\left(1-\Theta_{1} B^{S}-\ldots-\Theta_{Q} B^{Q S}\right)=$ seasonal moving average(MA) polynomial of order $Q$,

$(1-B)^{d}$ and $\left(1-B^{S}\right)^{D}=$ polynomial for ordinary and seasonal differencing respectively,

$Z_{t}=$ value of the series at time $t$, and

$a_{t}=$ white noise with zero mean and variance $\sigma^{2}$.

Estimating ARIMA model parameters (e.g., $p, d$, and $q$ etc.) and checking diagnostics are carried out in $R$-Project. After examining the ACF (Autocorrelation Function) and PACF (Partial Autocorrelation Function), a set of alternative models are identified with different model orders $p, d$, and $q$. To determine the best model among the alternatives, the diagnostics, and Akaike Information Criteria (AIC) are used. Finally, the model with good diagnostics and low AIC value is chosen. If the data generation process is stable, ARIMA models will provide reasonably accurate predictions. However, the basic ARIMA models would be inadequate to capture any abrupt shifts to new regimes. For instance, if an $\operatorname{ARIMA}(1,0,2)$ process $Z_{t}=\phi_{1} Z_{t 1}+\mu\left(1-\phi_{1}\right)+a_{t}-\theta_{1} a_{t-1}-\theta_{2} a_{t-2}$ is fit to the data and the process mean shifts to

new level $\mu_{n e w}$, then the one-step-ahead predictions will be consistently biased by an amount equal to $E[e]=\left(\mu_{\text {new }}-\mu\right)\left(1-\phi_{1}\right)+Z_{t-1}\left(\phi_{1 \text { new }}-\phi_{1}\right)+a_{t-1}\left(\theta_{1 \text { new }}-\theta_{1}\right)+a_{t-2}\left(\theta_{2 n e w}-\theta_{2}\right)$. Hence, EM and RLS are utilized to update these parameters after each observation. Time series model fitting for the proposed method as well as testing are given in section in 3 .

\subsection{Expectation maximization algorithm}

The Expectation Maximization (EM) algorithm is often used to estimate the parameters of mixtures or models with latent variables (Dempster et al. (1977)). In this paper, it is utilized for updating the mean of the process. The notation for EM is adopted from Hastie et al. (2009). Given $N$ sample points that are generated by a mixture of two distributions Eq. (5), the EM algorithm can converge to the 
parameters of $\boldsymbol{\theta}=\left[\theta_{1}=\left(\mu_{1}, \sigma_{1}\right), \theta_{2}=\left(\mu_{2}, \sigma_{2}\right), \pi\right]$. The first step of the EM algorithm specifies initial values for the parameters. In the expectation step, the algorithm computes the responsibilities $\gamma_{i}$ (i.e., the probability of an observation belonging to $Y_{2}$ ) for each data point. Using the calculated responsibilities, it then computes the five parameters in the maximization step. The iterations continue until the likelihood function convergences. The convergence of a basic EM algorithm is slow; however, there are techniques for accelerating the convergence (McLachlan and Peel (2000)). Basic equations pertaining to the EM for 2-component mixture model are given below. First, the probability density of $Y$ is written as a mixture in Eq. (6):

$$
Y=(1-\Delta) Y_{1}+\Delta Y_{2}
$$

where $Y_{1} \sim N\left(\mu_{1}, \sigma_{1}^{2}\right), Y_{2} \sim N\left(\mu_{2}, \sigma_{2}^{2}\right)$, and $\Delta \in 0,1$ with $\mathrm{P}(\Delta=1)=\pi$.

$$
g_{Y}(y)=(1-\pi) \phi_{\theta_{1}}(y)+\pi \phi_{\theta_{2}}(y)
$$

Where $\phi_{\theta}(x)$ denotes normal density. For a data set of $N$ points the loglikelihood function can be written as follows:

$$
l(\theta, Z)=\sum_{i=1}^{N} \ln \left[(1-\pi) \phi_{\theta_{1}}\left(y_{i}\right)+\pi \phi_{\theta_{2}}\left(y_{i}\right)\right]
$$

Where $\boldsymbol{\theta}=\left[\theta_{1}=\left(\mu_{1}, \sigma_{1}\right), \theta_{2}=\left(\mu_{2}, \sigma_{2}\right)\right.$, and $\left.\pi\right]$ and $Z$ represent the data points. Analytical maximization of Eq. (7) is difficult, however, if the observation is known to belong to $Y_{2}$ (i.e., with latent variable $\Delta_{i}=1$, otherwise $\Delta_{i}=0$ ), the loglikelihood can be written as in Eq. (8) and $\Delta_{i}=1$ s can be estimated by Eq. (9).

$$
\begin{gathered}
l(\theta ; \Delta, Z)=\sum_{i=1}^{N}\left[\left(1-\Delta_{i}\right) \ln \left[(1-\pi) \phi_{\theta_{1}}\left(y_{i}\right)\right]+\Delta_{i} \ln \left[\pi \phi_{\theta_{2}}\left(y_{i}\right)\right]\right] \\
\gamma_{i}(\theta)=E\left(\Delta_{i} \mid \theta, Z\right)=P\left(\Delta_{i}=1 \mid \theta, Z\right) \\
\hat{\mu}_{t+1}=\sum_{i=t-2}^{t}\left[\hat{\mu}_{1}\left(1-\gamma_{i}\right)+\hat{\mu}_{2} \gamma_{i}\right] / 3
\end{gathered}
$$

Given $N$ data points that are assumed to be generated by mixture of two distributions (i.e., normal and abnormal speeds), the EM algorithm is applied to determine the distribution parameters and responsibilities. Number of mixtures could be decided based on another algorithm as in (Melnykov and Melnykov (2012)). $N$ data points constitute the main input to the algorithm. The impact of sample size on prediction performances of EM algorithm is discussed in (Comert and Bezuglov (2013)). In the numerical examples, $N=10$ sample normal speed data points are used to represent the expected behavior and the most recent three observations are input for the real-time status. Other $N$ sizes are experimented, $N=10$ is found to be reasonable when EM run at each time step is considered. Initial parameter values are selected as significantly different two mixtures (e.g., $\hat{\boldsymbol{\theta}}=\left[\theta_{1}=\left(\mu_{1}=10, \sigma_{1}=10\right)\right.$ 
mph, $\left.\left.\theta_{2}=\left(\mu_{2}=80, \sigma_{2}=5\right) \mathrm{mph}, \pi=0.5\right]\right)$. Converging parameters are not experienced to be sensitive to these initial estimates as number data points is only 10. The EM algorithm provides the real-time estimation of the process mean at each time point from Eq. (10) which is subsequently used in OCPB, $\mathrm{OCPBr}$, and EM-ARIMA models for prediction.

\subsection{Recursive least squares filter}

Recursive least squares (RLS) is an algorithm that desired measures with errors are filtered in a specific system design (Wang et al. (2009); Paleologu et al. (2008); Bhotto and Antoniou (2013)). In the proposed method, RLS is applied to change $\operatorname{ARIMA}(1,0,2)$ model parameters (i.e., $\mu, \phi, \theta_{1}$, and $\left.\theta_{2}\right)$ for all state-by-state fitted models to filter the minor changes within a given state. Shown in Eq. (11), RLS contains design parameters of initial state covariance matrix $\mathbf{P}_{t}$ and forgetting factor $\lambda_{o}$ (Zeng and De Callafon (2006)). RLS is utilized to change ARIMA parameters based on recursive calculations and forecasting errors within $\min ( \pm 0.1,0.99)$ or $\max ( \pm 0.1,-0.99)$ intervals. Hence, parameter values change within $(-0.99,0.99)$.

$$
\begin{aligned}
\mathbf{K}_{t}=\frac{\lambda^{-1} \mathbf{P}_{t-1} \mathbf{X}_{t}}{1+\lambda^{-1} \mathbf{X}_{t}^{T} \mathbf{P}_{t-1} \mathbf{X}_{t}} & \text { Gain } \\
\boldsymbol{\alpha}_{\boldsymbol{t}}=\hat{\mathbf{X}}_{t}-\mathbf{X}_{t}^{T} \mathbf{w}_{t-1} & \text { Update } \\
\mathbf{w}_{t}=\mathbf{w}_{t-1}+\mathbf{K}_{t} \boldsymbol{\alpha}_{t} & \text { State Transition } \\
\mathbf{P}_{t}=\lambda^{-1} \mathbf{P}_{t-1}-\lambda^{-1} \mathbf{K}_{t} \mathbf{X}_{t}^{T} \mathbf{P}_{t-1} & \text { State Covariance Update } \\
e_{t}=\hat{Z}_{t}-Z_{t} & \text { Prediction Error } \\
\lambda_{t}=\lambda_{o} \lambda_{t-1}+1-\lambda_{o} & \text { Forgetting Factor Update }
\end{aligned}
$$

Where, $\boldsymbol{\alpha}_{\boldsymbol{t}}=\left(\mu, \phi_{1}, \theta_{1}, \theta_{2}\right), \mathbf{X}_{t}=\left(1, Z_{t-1}, e_{t-1}, e_{t-2}\right)$.

\section{Numerical experiments}

In this section, numerical results are presented for the performance of the proposed OCPBr model. The section contains training of the components and testing of the method against various other forecasting models which are briefly introduced in Table 1. The numerical experiments are performed on two datasets independently. Both datasets contain traffic speed data collected at several locations over multiple days. Traffic speed from loop dataset collected by the California PATH (Partners for Advanced Transit and Highways) program on I-880 for the Freeway Service Patrol Project and INRIX data from Norfolk and Hampton, Virginia are used. PATH database contains travel speeds, occupancies, and traffic volumes from 170 loop detectors at every 30 second collected over 46 weekdays. The loop data were collected in the morning (5:00 am to 10:00 am) and afternoon periods (2:00 PM to 8:00 PM). This is explicitly 
indicated in notations. The dataset also contains detailed records of all incidents including incident type, location, severity, and duration were kept for the data collection period (e.g., see Table 2). The detector sets and days are selected based on the incident records and the closest loop detectors reported to the location. F16PML4 represents February 16, PM part, and loop detector set of 4.

Table 1. Methods compared in the study

\begin{tabular}{|c|c|}
\hline Method & Description \\
\hline ARIMA & Simple ARIMA $(1,0,2)$ models trained on M2AM/PML4 and retrained on J1-0 and ARIMA $(1,1,1)$ model trained on M2AM/PML4 \\
\hline SARIMA & Seasonal ARIMA $(1,0,2)(1,1,0)$ and $\operatorname{ARIMA}(1,0,2)(1,0,0)$ models trained on M2AM/PML4 respectively \\
\hline EM-ARIMA & Mean adaptive $\operatorname{ARIMA}(1,0,2)$ model with EM algorithm \\
\hline ARIMA* & Simple ARIMA $(1,0,2)$ models refit on every loop detector on M2AM/PMLAll \\
\hline EM-ARIMA* & Mean adaptive ARIMA $(1,0,2)$ models with EM algorithm refit on every loop detector on M2AM/PMLAll \\
\hline MA & Five step moving average model \\
\hline WN & White Noise model \\
\hline $\mathrm{OCPB} m$ & Switching EM-ARIMA $(1,0,2)$ models with $m=4,5,6$-state HMM \\
\hline $\mathrm{OCPBr} m$ & Switching EM-ARIMA $(1,0,2)$ models and RLS filtering for ARIMA parameters with $m=4,5,6$-state HMM fit on F16PML4 \\
\hline $\mathrm{OCPBr}$ & Switching EM-ARIMA $(1,0,2)$ models and RLS filtering for ARIMA parameters with 5-state HMM and RLS fit on F16PML4 \\
\hline OCPBr* & $\begin{array}{l}\text { Switching EM-ARIMA }(1,0,2) \text { models and RLS filtering for ARIMA parameters with 5-state HMM and RLS fit on INRIX data } \\
\text { J13-0,1,5 }\end{array}$ \\
\hline LSTAR & Logistic Smooth Transition AutoRegressive model LSTAR $(2,4,4)$ fit on M3AM/PML4 \\
\hline SETAR & Self-exciting threshold autoregressive model SETAR $(2,4,4)$ fit on M3AM/PML4 \\
\hline NNTS & Neural Network nonlinear autoregressive model 5 dimensional NN fit on M3AM/PML4 \\
\hline AAR & Additive nonlinear autoregressive model with 5 nonparametric splines fit on M3AM/PML4 \\
\hline
\end{tabular}

Table 2. Example of lane blocking accidents in the data

\begin{tabular}{lcccc}
\hline Date & Start Time & End Time & Detector Set & Duration \\
\hline 16-Feb & $6: 48: 00 \mathrm{AM}$ & $9: 24: 00 \mathrm{AM}$ & 5 & $2: 38: 00$ \\
16-Feb & $3: 58: 00 \mathrm{PM}$ & $6: 29: 00 \mathrm{PM}$ & 4 & $2: 31: 00$ \\
18-Feb & $6: 52: 00 \mathrm{AM}$ & $6: 59: 00 \mathrm{AM}$ & 4 & $0: 06: 00$ \\
18-Feb & $7: 35: 00 \mathrm{AM}$ & $7: 44: 00 \mathrm{AM}$ & 4 & $0: 09: 00$ \\
18-Feb & $8: 57: 00 \mathrm{AM}$ & $9: 16: 00 \mathrm{AM}$ & 4 & $0: 19: 00$ \\
3-Mar & $7: 07: 00 \mathrm{AM}$ & $8: 21: 00 \mathrm{AM}$ & 4 & $1: 14: 00$ \\
3-Mar & $7: 04: 00 \mathrm{AM}$ & $9: 28: 00 \mathrm{AM}$ & 17 & $2: 24: 00$ \\
\hline
\end{tabular}

INRIX dataset is 20-second probe data collected from various freeway sections in Norfolk and Hampton, Virginia between 12:00 am-12:00 pm. In numerical experiments, no AM-PM separation is used for INRIX data. Fig. 2 demonstrates the utilized freeway sections that are given as 110+04870, 04871, 04872, 04873, 04874, 04875, and 04876. These are actual codes from the dataset for these freeway sections. For brevity, they are referred using the last digits and locations: $0,1,2,3,4,5$, and 6 in Table 3 with section 
lengths of $0.154,0.327,0.605,0.708,1.543,0.273$, and 0.884 miles respectively. The part of the dataset used in this paper is 19 weekdays and 7 different freeway sections from January 2014 which are coded as day and location (section), for instance, $J 1-0$ represents January 1 and location 0 . There is no AM or PM mark in INRIX data as it is completed over complete days.

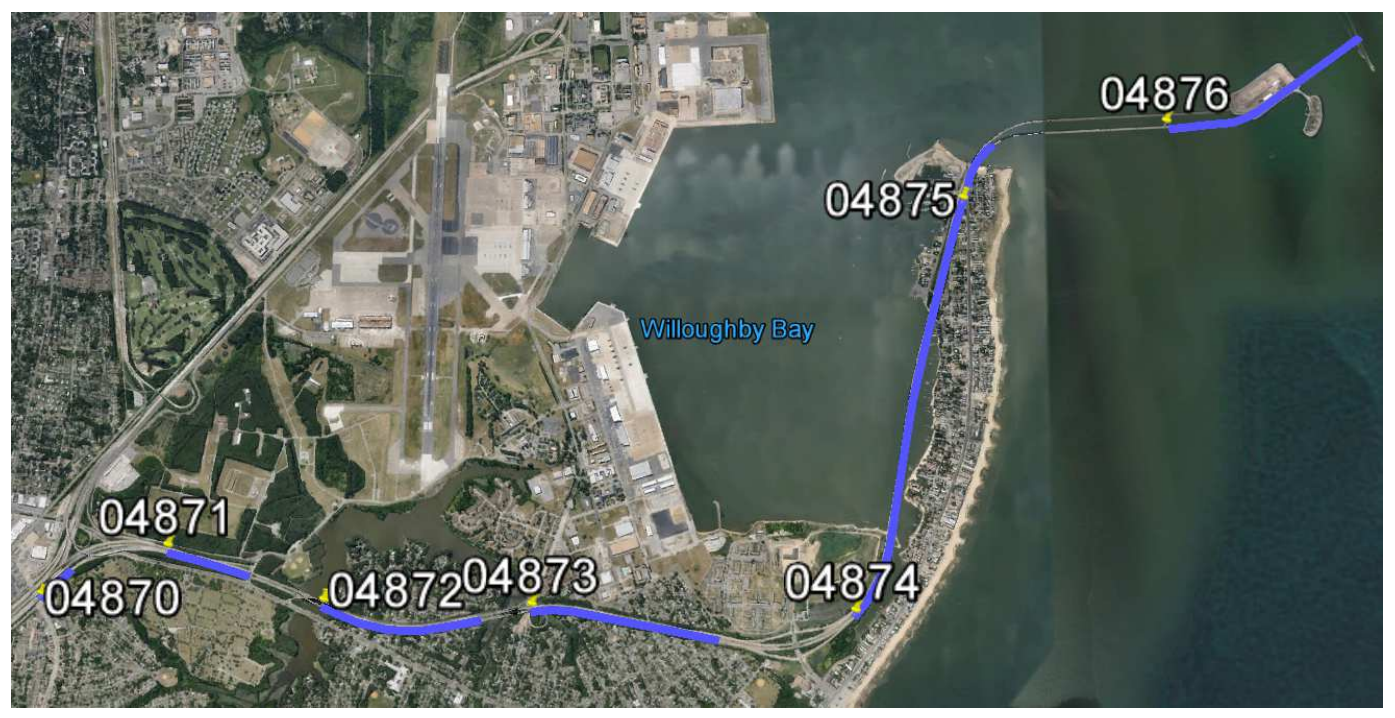

Fig. 2. Freeway sections used from INRIX data

Table 3 lists the total of 41 days of 1-minute aggregated travel speed data that are used for model building and testing from different locations. Loop detector numbers closest to the incidents and impacted locations in INRIX data (i.e., shown by * next to location number) are also given in the table. The testing of all models is performed on days with no incident occurring near these loops and locations and on days with accidents. Broadly, changes occurred on the testing days are also shown in the table. Although the changes do not show uniform effects on different days, in order to have a better idea about the performance of the proposed model they are given considering the drop of average speed to different states: (1) Major Accident-Major Change (10 miles per hour (mph)), (2) Minor Accident-Minor Change (20 mph), (3) Instability-Minor (40 mph), (4) Instability-Major (50 mph), and (5) Normal Driving (60 mph). Speed predictions for loop data are carried out separately for AM and PM. For INRIX data, they are done daily since samples are 24 hours from weekdays. Selection of these states are explained in subsection 2.1.

The normality assumption of DGP is tested with Shapiro Wilk in $R$ for state-by-state sections of datasets where $p$ values are presented in Table 4 . More than half of the tests reject (i.e., $p \leq 0.05$ ) the null hypothesis that the data sections from different states are normally distributed. The normal probability plots are also checked. Overall, the samples look approximately normal. The main effect of normality is on EM and HMM models where the DGP is assumed to be mixtures of normal distributions. Probability of states given the observations are calculated from Normal distributions. Although, time 
Table 3. Data used in numerical experiments, purpose of use and type of change the day contains

\begin{tabular}{|c|c|c|c|c|c|}
\hline \multicolumn{3}{|c|}{ Loop Data } & \multicolumn{3}{|c|}{ INRIX Data } \\
\hline Day & Usage & Changes & Day & Usage & Changes \\
\hline F16AML4/F16PML4 ${ }^{a}$ & OCPB(ARIMA) fit & Major & $\mathrm{J} 1-0^{b}$ & HMM train/ARIMA fit & Major \\
\hline M2AML4/M2PML4 & ARIMA fit & No & $\mathrm{J} 13-0^{*}, 1^{*}, 5$ & OCPB*(ARIMA) fit & Major \\
\hline M2AMLAll/M2PMLAll & ARIMA* fit & No & $\mathrm{J} 17-3^{*}$ & HMM train & Major \\
\hline M3AML4/M3PML17 & HMM train/ARIMA fit & Major & $\mathrm{J} 1-1,2,3,4,5,6$ & testing & No \\
\hline F17AML5/F17PML5 & testing & No/Major & $\mathrm{J} 2-0,1,2,3,4,5,6$ & testing & No \\
\hline F18AML4/F18PML12 & testing & Major/Major & J3-0,1,2,3*,4,5,6 & testing & Minor \\
\hline F19AML4/F19PML19 & testing & No/Major & $\mathrm{J} 6-0,1,2,3,4,5^{*}, 6$ & testing & Major \\
\hline F22AML12/F22PML12 & testing & No/Major & $\mathrm{J} 7-0,1,2,3,4^{*}, 5,6$ & testing & Minor \\
\hline F23AML2/F23PML4 & testing & Major/Major & J8- $0,1,2,3,4,5,6$ & testing & No \\
\hline F24AML7/F24PML7 & testing & No/Minor & J9- $0,1,2,3,4,5,6$ & testing & No \\
\hline F25AML4/F25PML13 & testing & No/Major & $\mathrm{J} 13-2^{*}, 3^{*}, 4,6$ & testing & Major \\
\hline F26AML12/F26PML12 & testing & No/Major & $\mathrm{J} 14-0,1,2^{*}, 3,4,5,6$ & testing & Major \\
\hline M1AML4,L7/M1PML4,L7 & testing & No/Minor-No & $\mathrm{J} 16-0,1,2,3,4,5,6$ & testing & No \\
\hline M4AML20/M4PML20 & testing & No/No & $\mathrm{J} 17-0^{*}, 1^{*}, 2^{*}, 4,5,6$ & testing & Major \\
\hline M5AML20/M5PML20 & testing & No/Major & J20-0,1,2,3,4,5,6 & testing & No \\
\hline M8AML3/M8PML3 & testing & Minor/No & $\mathrm{J} 21-0,1,2,3,4^{*}, 5,6$ & testing & Major \\
\hline M9AML16/M9PML16 & testing & Minor/No & $\mathrm{J} 22-0,1,2,3,4,5^{*}, 6$ & testing & Major \\
\hline M10AML1/M10PML1 & testing & No/No & $\mathrm{J} 24-0,1,2,3,4^{*}, 5,6$ & testing & Major \\
\hline M11AML16/M11PML16 & testing & No/No & $\mathrm{J} 27-0,1,2,3,4,5,6$ & testing & No \\
\hline M12AML19/M12PML19 & testing & No/Major & $\mathrm{J} 31-0^{*}, 1^{*}, 2^{*}, 3^{*}, 4^{*}, 5^{*}, 6$ & testing & Major \\
\hline M15AML4/M15PML18 & testing & Major/No & & & \\
\hline M16AML12/M16PML12 & testing & No/Major & & & \\
\hline M17AML5/M17PML5 & testing & No/Major & & & \\
\hline M18AML4,L18/M18PML4,L18 & testing & Major/Minor-No & & & \\
\hline M19AML4,L19/M19PML4,L19 & testing & No/Major & & & \\
\hline
\end{tabular}

series models are also assuming Gaussian errors, closely normal process can be reasonable to assume for time series modeling where normal errors are important for maximum likelihood estimators of parameter estimators. Even though the normality is partially supported by the tested samples in this paper, the assumption may not always hold in practice (Park et al. (2010); Zou (2013)).

Table 4. Shapiro-Wilk test for normality F16PML4 and J13 (locations)

\begin{tabular}{lccccc}
\hline State & p-val & s size & State & p-val & s size \\
\hline 1 & 0.727 & 53 & $1(0)$ & $<0.001$ & 61 \\
2 & 0.136 & 85 & $2(1)$ & $<0.024$ & 50 \\
3 & 0.658 & 23 & $3(1)$ & $<0.023$ & 21 \\
4 & 0.269 & 26 & $4(5)$ & $<0.001$ & 145 \\
5 & $<0.001$ & 167 & $5(0)$ & $<0.001$ & 1198 \\
\hline
\end{tabular}




\subsection{Model training}

In this section, trainings of the method components are presented. EM algorithm only requires initial parameter estimates, historical, and current observations, it does not require any training. Thus, discussion on HMM, ARIMA, and RLS are provided in this subsection. Training of the HMM also contains the optimum number of states analysis. For the proposed method, OCPBr denotes the model trained on the loop data and OCPBr* is the method retrained using INRIX data(see Table 1).

\subsubsection{HMM and number of states}

In HMM model formulae from subsection 2.1, the state variable $X_{t}$ can be discrete or continuous. When it is discrete, the number of states in the model can be determined manually based on the application or algorithmically (Celeux and Durand (2008)). In this work, states are discrete and they represent the road conditions that the model can recognize. As an example Fig. 3 illustrates the outputs of five state HMM on the two datasets, where the states are: (1) Constrained-Major Accident, (2) ConstrainedMinor Incident, (3) Transitive-Instability, (4) Unconstrained-higher flow, and (5) Unconstrained traffic: $X_{t}=\{1,2,3,4,5\}$. Probability distribution $P\left(X_{t} \mid X_{t-1}\right)$ represents the change points or state transition probabilities. Number of states can be viewed as traffic regimes such as unconstrained stable flow with close to free flow speeds and constrained traffic with several incident types tire blast, without lane blockage, with lane/lanes blockages. For instance in Fig. 3, a 5-state HMM can detect five distinct road conditions such as state 5 and 4 which represent stable flow at high speeds. State 4 might be at higher flow rates reflecting close but less than 5-10 mph speed values compared to state 5 . State 3 is rather transitive state either from states 1 and 2 to 4 and 5 or from 4 and 5 to 2 and 1 . State 1 and 2 might represent instable flow conditions with incident, congestion, queue forming and discharging, and constrained traffic flow with low speeds 5-15 mph and 20-30 mph respectively. The analysis is carried on two datasets independently. Both datasets contain 1-minute traffic speed data collected at several locations over multiple days. Dataset 1 (Loop Data) used has a total of 14 days from 5 loop locations (i.e., excluding the additional loop data utilized for further testing). Dataset 2 (INRIX data) that is selected for numerical examples has 17 days from 7 locations.

There are a few observed differences between the two datasets. The loop data used contain many changes (i.e., magnitude and duration that can be seen from Table 2), however, INRIX data do not have as many of such instances (or reflect to that extend) except few days with change points over shorter time periods which can be seen in Fig. 3. Average speeds in INRIX data are given as integer values where loop data express the observations with six decimal places. Noted also by Kim and Coifman (2014), INRIX data repeat observations if no vehicle reports. This contributes to less variation and almost filtered behavior. Finding the optimal number of states, i.e., dimensionality of the model, is essential for its 

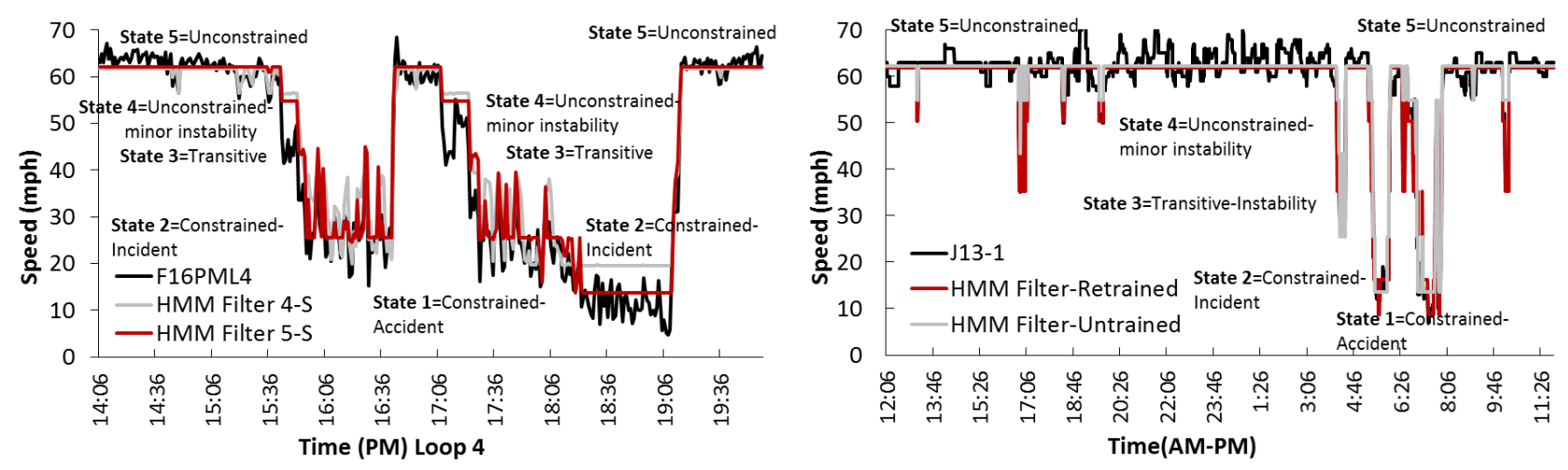

Fig. 3. Examples of HMM filtering of F16PML4 and INRIX J13-1 showing different states of the process

performance and robustness. Models with higher number of states typically demonstrate better fit to observed data, however, they may over-fit and fail to generalize on unseen data. In this work, the authors studied the optimal number of states (dimensionality) of HMMs by testing model's Akaike and Bayesian information criteria (AIC and BIC) on training and test data. AIC and BIC are evaluated using formulae $\mathrm{AIC}=2 \cdot k-2 \cdot \ln (L)$, where $n$ is the size of the training dataset, $L$ is likelihood of the model, $k$ is number of free model parameters, which is approximately the square of the number of states in case of HMMs. For $\mathrm{BIC}=\ln (n) \cdot k-2 \cdot \ln (L)$. Both criteria penalize the models for extra complexity and as such, the best fit HMM is expected to minimize AIC and BIC. In addition, impact of the number of states on the forecasting performances are also investigated to find out recommended number of states based on the analyzed datasets.

Dataset 1 is split into two subsets one for fitting ( 7 days AM/PM:F16L4,F18L4, F19L4, F23L4, F24L7, F25L4, F26L12) and one for testing (7 days AM/PM:M01L4, M5L20, M15L4, M16L12, M17L5, M18L18, M19L4) of HMMs. Models with different number of states are fit using the training dataset and their AIC and BIC criteria are recorded. As it is illustrated by Fig. 4a, both criteria are quite similar with the recommended model size. After a rapid decrease from 2-state to 5-state models, both graphs do not significantly change, suggesting that the 5 -state model is optimal for Dataset 1 . Fig. $4 \mathrm{~b}$ demonstrates AIC and BIC of the models on the testing subset of Dataset 1 (not previously seen by the models). In the figure, the values decrease quickly from 2-state to 5-state models and then the decrease slows down. Additional states after 5-state results in increasing AIC and BIC values.

In case of Dataset 2, for number of states analysis again the HMMs are trained and tested using the same locations. Similarly to the previous case, for each location, the dataset is split into training and testing subsets, which are designated as $J 1, J 2, J 3, J 6, J 7, J 8$ for training and $J 9, J 13, J 14, J 16$, $J 17, J 20, J 21, J 22, J 24, J 27$, and $J 31$ for testing at all locations 0 to 6 in Table 3. Fig. 5 shows AIC and BIC of the models trained on Dataset 2 from locations 0 to 6 . Unfortunately, due to the differences 


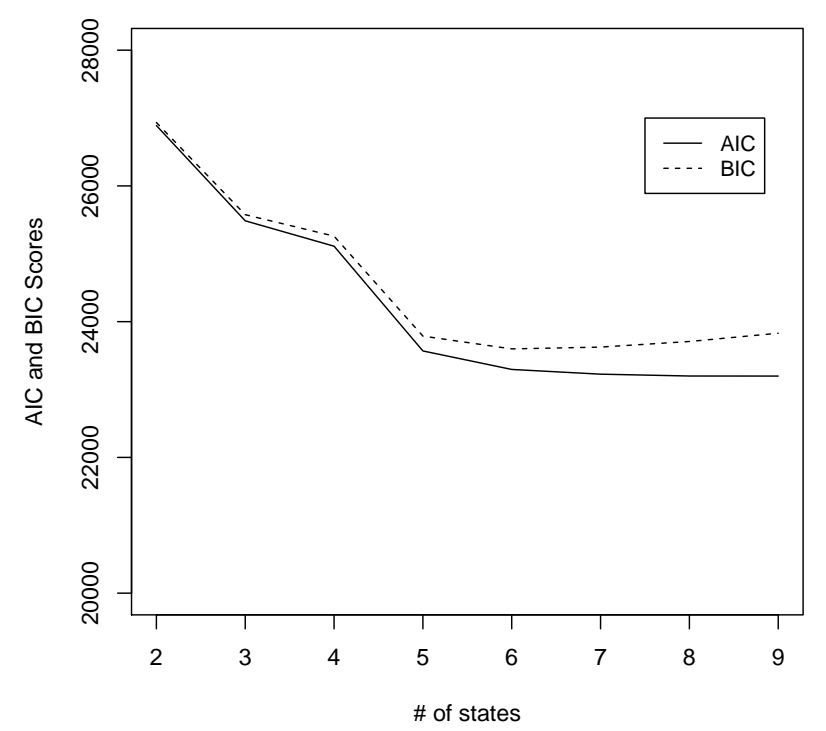

(a) AIC and BIC for training data

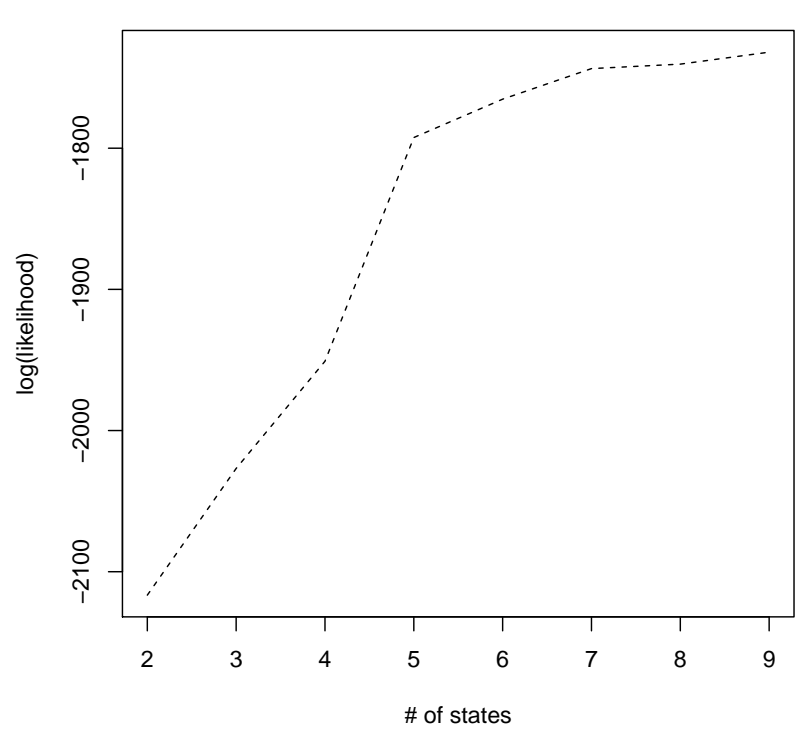

(b) AIC and BIC for testing data

Fig. 4. HMM performance on dataset 1 (training and testing datasets)

mentioned earlier, it is found to be difficult to determine one single model size to perform the best in all cases. However, both criteria suggest that the optimum dimensionality between 3 and 7 states:

- 3-state HMM is slightly preferred in location 2 and 5.

- 5-state HMM is recommended for locations 1 and 3.

- 6-state HMM can be used for location 4.

- 7-state HMM can be used for location 0.

The performance of the models on Dataset 2 is illustrated in Fig. 5. Performance of the models containing more than 6 states generally deteriorate except for the model that is fit at location 0 as AICs and BICs seen in the figure. Even though the 5-state HMM does not always have the lowest AIC or BIC scores, it can generalize to unseen data very well. In the literature, five component mixture model for speed data is also selected by Park et al. (2010). Fig. 6 summarizes how well the models can generalize to unseen (testing) data. In the figure, the 5-state models, which are fit to the data at locations 1 and 3 , demonstrate the best results overall. Throughout the paper the proposed prediction model containing HMM1 (5-state HMM trained on M3PML17) and HMM2 (5-state HMM trained on J17-3) are denoted as $\mathrm{OCPBr}$ and $\mathrm{OCPBr} *$ respectively.

As with most local optimization algorithms, the key to success is proper initialization. However, 


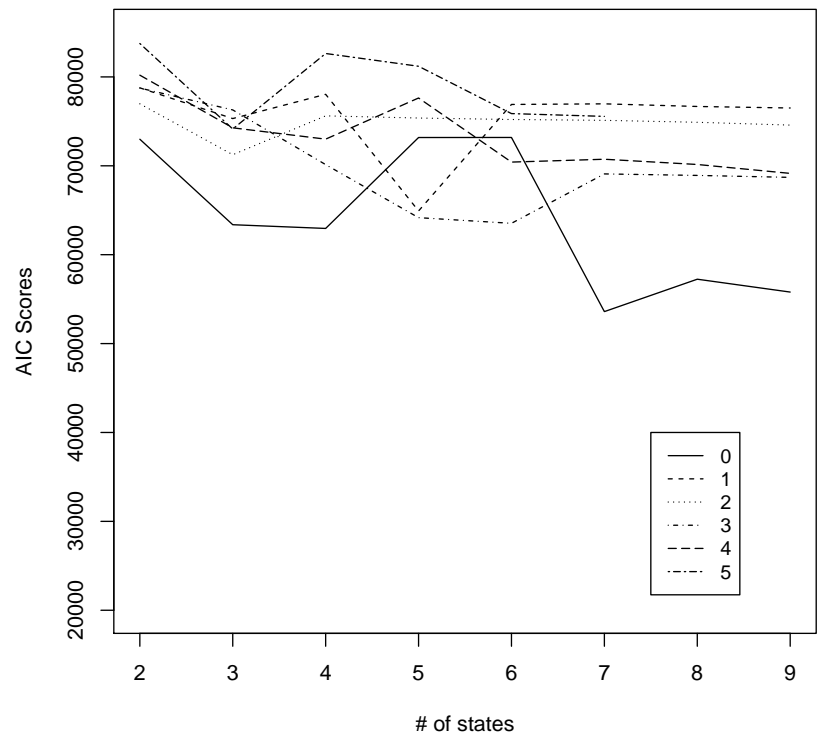

(a) AIC

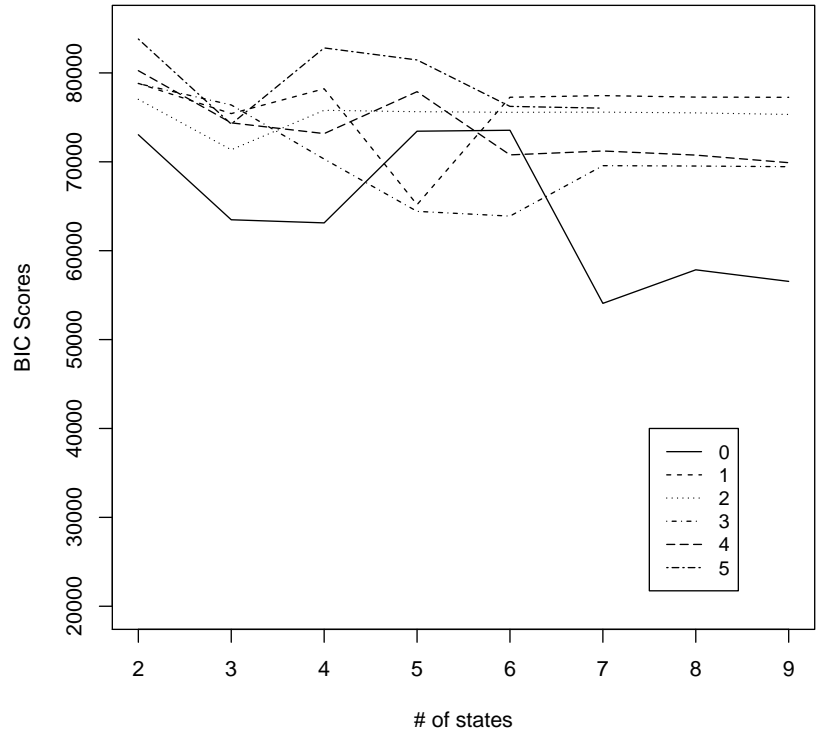

(b) BIC

Fig. 5. AIC and BIC on training Dataset 2 (INRIX)

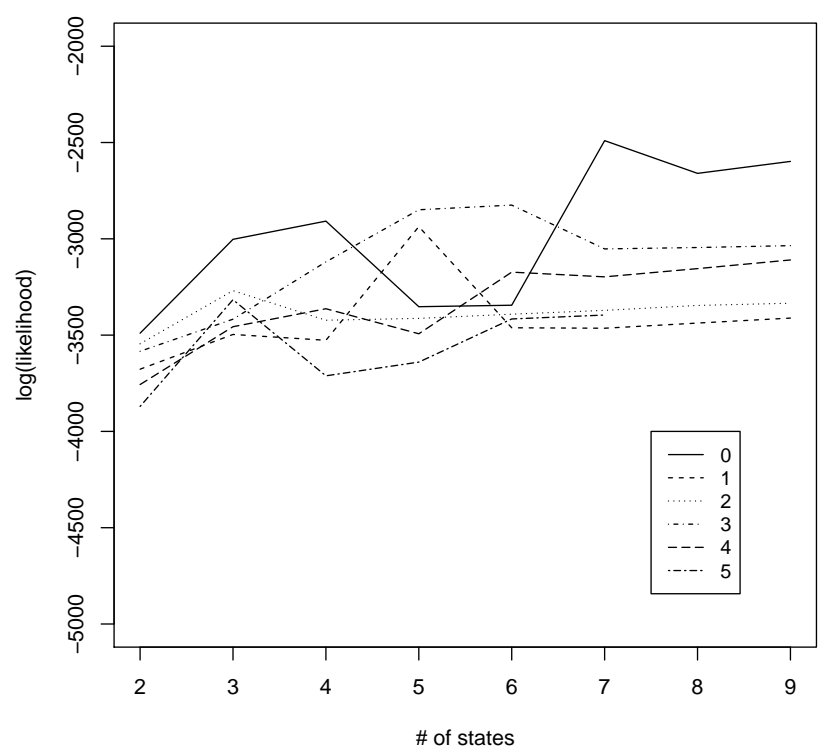

Fig. 6. AIC and BIC on unseen (testing) INRIX dataset

optimized initialization of HMM training is beyond the scope of the paper. The initial HMM parameters in this study are set to $10 \mathrm{mph}$ for Major Accident and $20 \mathrm{mph}$ for Minor Accident state 1 and state 2 respectively constrained flow stages, state $340 \mathrm{mph}$ for Major Instability or transitive or queue discharge 
state, state $450 \mathrm{mph}$ for Minor Instability in an unconstrained flow, and state $560 \mathrm{mph}$ for Normal Driving in an unconstrained flow (see Table 5). The choice of the initial mean values in the table facilitates the interpretation of the model and enables to relate the state definitions after the trained HMM. Converged parameters for both HMM models are given in Table 5.

Table 5. HMM parameters trained on M3PML17 and J17-3

\begin{tabular}{lccc}
\hline State & Initial $\left(\mu, \sigma^{2}\right)$ & HMM 1 $\left(\mu, \sigma^{2}\right)$ & HMM 2 $\left(\mu, \sigma^{2}\right)$ \\
\hline 1 & $(10,1)$ & $(13.65,5.50)$ & $(8.77,2.60)$ \\
2 & $(20,1)$ & $(25.55,24.97)$ & $(16.14,4.47)$ \\
3 & $(40,1)$ & $(43.34,3.36)$ & $(35.32,28.50)$ \\
4 & $(50,1)$ & $(54.81,19.29)$ & $(50.45,2.07)$ \\
5 & $(60,1)$ & $(62.19,2.31)$ & $(61.96,4.23)$ \\
\hline
\end{tabular}

Table 6 shows state transition matrices $P\left(X_{t} \mid X_{t-1}\right)$ of the 5-state HMM models that are trained on M3PML17 and J17-3. Models are conservative as the probability of staying at the same state is very high for major accident, minor incident, instabilities, and normal driving (i.e., $P\left(X_{t}=k \mid X_{t-1}=k\right)=97.5 \%$, $92.7 \%, 90.1 \%, 86.2 \%, 99.2 \%)$ and $(97.9 \%, 95.5 \%, 86.8 \%, 81.8 \%, 99.8 \%)$ respectively for two datasets. Only staying at instabilities are relatively lower $90.1 \%, 86.2 \%$ and $86.2 \%, 81.8 \%$.

Table 6. $P\left(X_{t} \mid X_{t-1}\right)$, HMM transition matrices trained on M3PML17 and J17-3

\begin{tabular}{lccccc|ccccc}
\hline \multicolumn{1}{c}{ HMM1 } \\
\hline State & 1 & 2 & 3 & 4 & 5 & 1 & 2 & 3 & 4 & 5 \\
\hline 1 & 0.975 & 0.025 & 0.000 & 0.000 & 0.000 & 0.979 & 0.010 & 0.011 & 0.000 & 0.000 \\
2 & 0.018 & 0.927 & 0.054 & 0.063 & 0.031 & 0.022 & 0.955 & 0.023 & 0.000 & 0.000 \\
3 & 0.000 & 0.059 & 0.901 & 0.039 & 0.000 & 0.000 & 0.079 & 0.868 & 0.026 & 0.026 \\
4 & 0.000 & 0.000 & 0.092 & 0.862 & 0.066 & 0.000 & 0.000 & 0.091 & 0.818 & 0.091 \\
5 & 0.000 & 0.000 & 0.000 & 0.008 & 0.992 & 0.000 & 0.000 & 0.001 & 0.001 & 0.998 \\
\hline
\end{tabular}

HMM models in OCPBr are used to find whether there is a change point at time $t$ where the trained HMM looks at five immediate preceding speed values $Z_{-1 .-5}=\left\{Z_{t-1}, Z_{t-2}, Z_{t-3}, Z_{t-4}, Z_{t-5}\right\}$ and applies the Forward-Backward algorithm (Rabiner (1989)) to find the posterior probability of each state at time $t: P\left(X_{t} \mid Z_{-1 . .-5}\right)$. The state $i$ that maximizes the predicted state $P\left(X_{t}=i \mid Z_{-1 . .-5}\right)$ is utilized to choose the proper speed forecasting model. To account for the potential effect of the number of historical data points for change point detection, HMMs are also trained on 10 and 20 minutes of data, but, no significant difference in the outcomes was found. Note that HMM models that are used in the proposed model are trained only on continuous sections of data sets loop data PM section-M3PML17 and INRIX data single day-J17-3. 


\subsubsection{ARIMA fitting}

For numerical examples, several ARIMA models including seasonal ARIMA (SARIMA) are fit to compare the prediction performance of the proposed method and to test its transferability and the impact of number states. A total of 65 (i.e., AM-PM) ARIMA models are obtained for forecasting which are listed as:

1. To compare with $\operatorname{OCPBr}$, two $\operatorname{ARIMA}(1,0,2)$, two $\operatorname{ARIMA}(1,1,1)$, a $\operatorname{SARIMA}(1,0,2)(1,1,0)$ with period $S=79$, and a SARIMA $(1,0,2)(1,0,0)$ with $S=79$ models are fitted to M2AM/PML4 and one $\operatorname{ARIMA}(1,0,2)$ is fitted to J1-0 which are all normal speed days (see Table 7).

2. For transferability, thirty eight $\operatorname{ARIMA}(1,0,2)$ are fitted to all 19 loop sets on M2AM/PM to compare proposed $\mathrm{OCPBr}$ and $\mathrm{OCPBr}^{*}$ models with loop-by-loop fit ARIMA* and EM-ARIMA*. Fitted models are given in Table 8.

3. In order to test the impact of number of states on forecasting performances, twenty models are fitted to each state in $\mathrm{OCPBr}-4, \mathrm{OCPBr}$ (Table 9), $\mathrm{OCPBr}^{*}$ (Table 10), and OCPBr-6 models. For this purpose, F16PML4 and J13-0,1,5 are used for ARIMA parameter estimation in the proposed OCPB models since they represent multiple changing states (see Fig. 3).

Table 7. Basic ARIMA and EM-ARIMA parameters for loop and INRIX data

\begin{tabular}{lcccccccc}
\hline Day & Models & $\phi$ & $\theta_{1}$ & $\theta_{2}$ & $\Phi$ & Intercept & PP Test & Box-Ljung Test \\
\hline M2AML4 & ARIMA(1,0,2) & 0.95 & 0.67 & 0.09 & - & 61.87 & $<0.01$ & 0.25 \\
M2PML4 & ARIMA(1,0,2) & 0.96 & 0.52 & 0.07 & - & 60.63 & $<0.01$ & 0.99 \\
M2AML4 & ARIMA $(1,1,1)$ & 0.70 & 0.97 & - & - & - & $<0.01$ & 0.10 \\
M2PML4 & ARIMA $(1,1,1)$ & 0.17 & 0.72 & - & - & - & $<0.01$ & 0.98 \\
M2AML4 & SARIMA(1,0,2)(1,1,0) & 0.88 & 0.19 & 0.05 & -0.57 & - & $<0.01$ & 0.90 \\
M2PML4 & SARIMA(1,0,2)(1,0,0) & 0.96 & 0.52 & 0.07 & 0.05 & 60.66 & $<0.01$ & 0.99 \\
J1-0 & ARIMA $(1,0,2)$ & 0.92 & -0.002 & -0.01 & - & 62.31 & $<0.01$ & 0.38 \\
\hline
\end{tabular}

In model fitting, a normal driving day, M2AM/PM are utilized separately and speed measurements are found to be stationary by observing the diagnostics. So, no differencing is needed. However, a formal treatment for the stationarity of the data segments is carried out with Phillips-Perron Unit Root (PP) Test with a null hypothesis of nonstationary process that is time series has a unit root (see Tables 7,9, and 10 for $p$ values). Model fitting observations on M2AM/PM and J1-0 are supported by all $p$ values $<0.01$ which reject the null for unit root tests. It can be observed from the Table 10 that state 3 in INRIX data is failed to reject ( $p$ value 0.43 ) with sample size of 21 data points in that segment and test for state 4 of loop data barely fails to reject with $p$ value of 0.06 . Normal driving days as well as all of the state-by-state models are fitted to $\operatorname{ARIMA}(1,0,2) . \operatorname{ARIMA}(1,0,2)$ model is empirically found to be 
Table 8. Parameters of $\operatorname{ARIMA}(1,0,2)$ models for all loops trained on M2AM/PML4

\begin{tabular}{l|cccccccc}
\hline & \multicolumn{9}{|c}{ AM } & \multicolumn{5}{c}{ PM } \\
\hline Loop & $\phi$ & $\theta_{1}$ & $\theta_{2}$ & Intercept & $\phi$ & $\theta_{1}$ & $\theta_{2}$ & Intercept \\
\hline 1 & 0.959 & 0.299 & 0.448 & 60.15 & 0.940 & 0.629 & 0.050 & 62.58 \\
2 & 0.981 & 0.844 & 0.026 & 58.53 & 0.890 & 0.791 & -0.011 & 58.42 \\
3 & 0.982 & 0.742 & 0.149 & 61.11 & 0.962 & 0.675 & 0.101 & 60.09 \\
4 & 0.847 & 0.199 & 0.037 & 61.10 & 0.956 & 0.521 & 0.065 & 60.61 \\
5 & 0.959 & 0.704 & 0.075 & 61.01 & 0.946 & 0.231 & 0.026 & 56.70 \\
6 & 0.978 & 0.829 & 0.088 & 61.03 & 0.974 & 0.797 & 0.095 & 59.99 \\
7 & 0.957 & 0.533 & 0.299 & 59.38 & 0.944 & 0.586 & 0.065 & 58.79 \\
8 & 0.966 & 0.626 & 0.035 & 59.52 & 0.978 & 0.618 & 0.110 & 57.53 \\
9 & 0.978 & 0.801 & 0.093 & 61.39 & 0.944 & 0.658 & 0.047 & 61.76 \\
10 & 0.977 & 0.823 & 0.009 & 61.52 & 0.989 & 0.225 & 0.268 & 55.99 \\
11 & 0.969 & 0.772 & 0.112 & 62.52 & 0.976 & 0.861 & 0.024 & 57.88 \\
12 & 0.846 & 0.101 & 0.080 & 61.63 & 0.951 & 0.283 & 0.076 & 51.86 \\
13 & 0.801 & 0.304 & 0.083 & 62.43 & 0.929 & 0.198 & -0.197 & 59.70 \\
15 & 0.964 & 0.583 & 0.179 & 62.18 & 0.916 & 0.178 & 0.110 & 60.18 \\
16 & 0.958 & 0.725 & 0.093 & 59.89 & 0.896 & 0.739 & -0.024 & 55.89 \\
17 & 0.758 & 0.042 & -0.129 & 61.80 & 0.954 & 0.504 & 0.127 & 61.74 \\
18 & 0.977 & 0.733 & 0.144 & 62.51 & 0.977 & 0.681 & 0.189 & 59.29 \\
19 & 0.856 & 0.660 & 0.045 & 64.30 & 0.822 & 0.176 & -0.213 & 62.42 \\
20 & 0.981 & 0.648 & 0.229 & 60.73 & 0.937 & 0.562 & 0.067 & 59.45 \\
\hline & & & & & & & &
\end{tabular}

Table 9. State-by-state ARIMA parameters for OCPBr trained on F16PML4

\begin{tabular}{c|cccccccc}
\hline Day & State & Models & $\phi$ & $\theta_{1}$ & $\theta_{2}$ & Intercept & PP Test & Box-Ljung Test \\
\hline PM & 1 & ARIMA(1,0,2) & 0.35 & 0.07 & 0.12 & 15.95 & $<0.01$ & 0.95 \\
PM & 2 & ARIMA(1,0,2) & -0.08 & -0.23 & -0.06 & 24.95 & $<0.01$ & 0.61 \\
PM & 3 & ARIMA(1,0,2) & -0.58 & -0.45 & 0.10 & 32.97 & $<0.01$ & 0.99 \\
PM & 4 & ARIMA (1,0,2) & 0.53 & 0.09 & 0.01 & 43.66 & 0.06 & 0.69 \\
PM & 5 & ARIMA(1,0,2) & 0.96 & 0.52 & 0.07 & 62.27 & $<0.01$ & 0.97 \\
\hline
\end{tabular}

Table 10. State-by-state parameters for OCPBr* trained on J13-0,1,5

\begin{tabular}{l|cccccccc}
\hline Time & State & Models & $\phi$ & $\theta_{1}$ & $\theta_{2}$ & Intercept & PP Test & Box-Ljung Test \\
\hline AM-PM & 1 & ARIMA $(1,0,2)$ & 0.42 & -0.44 & 0.14 & 8.31 & 0.02 & 0.96 \\
AM-PM & 2 & ARIMA $(1,0,2)$ & 0.32 & -0.28 & 0.01 & 15.79 & 0.02 & 0.92 \\
AM-PM & 3 & ARIMA(1,0,2) & 0.37 & -0.14 & -0.11 & 28.13 & 0.43 & 0.81 \\
AM-PM & 4 & ARIMA (1,0,2) & 0.81 & 0.07 & 0.05 & 51.62 & $<0.01$ & 0.55 \\
AM-PM & 5 & ARIMA(1,0,2) & 0.87 & 0.01 & -0.02 & 61.97 & $<0.01$ & 0.66 \\
\hline
\end{tabular}

one of the best candidates with low AIC values, no clear patterns for standard residuals, and $p$ values are consistently greater than 0.05 for the Box-Ljung statistics (up to 10 step) which support independence 
of residuals (see Column 9 of Table 7). For SARIMA models, from autocorrelation function, lag 16 is observed to be higher barely than threshold values $( \pm 0.1)$, which shows weak seasonality. So, period values of $4,8,12,16,32, \ldots, 192$ are investigated. None of which were able to pass Box-Ljung test. Next close values are tried and period=79 were able to provide good and passing diagnostics (Wei (2006)). In the Table 7, all of the Box-Ljung Test $p$ values are larger than 0.05 significance level, that is, failing reject the null hypothesis of independent residuals. Optimum ARIMA model fitting for each state is also interesting research topic, however, it is beyond the scope of this paper. Employing the same ARIMA model can be desirable as the model orders remain the same when parameters are changing that simplifies the recursive filtering. For state-by-state ARIMA fitting, HMM is used to filter the training days and segments of the same states are linked together. The impact of mixing on the model fitting is relatively limited as both training days (i.e., F16PML4 and J13) consist of long runs of particular states and only single day of data from each datasets is used for model building. Estimated parameter values for 5-state OCPBr and OCPBr* models are given in Tables 9 and 10. Similarly, 4 and 6 state models are derived and used in numerical examples.

For speed forecasts using time series models, $\operatorname{AR}(\infty)$ representation in Eq. (12) is adopted which provides recursive calculation of coefficients that weighs past observations. Using Eq. (12), one can obtain the forecasting formula for $\operatorname{ARIMA}(1,0,2)$ as in Eq. (13). In Eq. (13), the Intercept $\mu$ and model parameters (e.g., $\phi$ and $\theta$ s) are kept constant in ARIMA and ARIMA* models. Similarly, ARIMA(1,1,1), $\operatorname{SARIMA}(1,0,2)(1,1,0)$, and $\operatorname{SARIMA}(1,0,2)(1,0,0)$ forecasts are calculated from Eqs. (14-16) respectively. EM-ARIMA and EM-ARIMA* forecasts are produced using Eq. (13) with incorporation of Eq. (10) for updating $\mu$ after each new speed observation received.

$$
\begin{gathered}
\psi(B)\left(Z_{t}-\mu\right)=a_{t} \psi(B)=\Phi(B) \phi(B)(1-B)^{d}(1-B)^{D} / \theta(B) \\
\hat{Z}_{t+1}=\left(1-\sum_{i=1}^{\infty} \psi_{i} \mu\right)+Z_{t} \sum_{i=1}^{\infty} B \psi_{i}
\end{gathered}
$$

Where $\psi_{0}=1, \psi_{1}=\phi-\theta_{1}, \psi_{2}=\psi_{1} \theta_{1}-\theta_{2}$, and $\psi_{i}=\psi_{i-1} \theta_{1}-\psi_{i-2} \theta_{2}$ for $i>2$.

$$
\hat{Z}_{t+1}=Z_{t} \sum_{i=1}^{\infty} B \psi_{i}
$$

Where $\psi_{0}=1, \psi_{1}=1+\phi-\theta_{1}, \psi_{2}=\psi_{1} \theta_{1}-\phi$, and $\psi_{i}=\psi_{i-1} \theta_{1}$ for $i>2$.

$$
\begin{gathered}
(1-\phi B)\left(1-\Phi B^{79}\right)\left(1-B^{79}\right)\left(Z_{t}-\mu\right)=\left(1-\theta_{1} B-\theta_{2} B^{2}\right) a_{t} \\
\hat{Z}_{t+1}=Z_{t} \sum_{i=1}^{\infty} B \psi_{i}
\end{gathered}
$$

Where $\psi_{0}=1, \psi_{1}=\phi-\theta_{1}, \psi_{2}=\psi_{1} \theta_{1}-\theta_{2}$, and $\psi_{i}=\psi_{i-1} \theta_{1}-\psi_{i-2} \theta_{2}$ for $i>2, i \neq 79,80,158,159$, $\psi_{i}=\psi_{i-1} \theta_{1}-\psi_{i-2} \theta_{2}+\Phi+1, i=79, \psi_{i}=\psi_{i-1} \theta_{1}-\psi_{i-2} \theta_{2}-\phi-\phi \Phi, i=80, \psi_{i}=\psi_{i-1} \theta_{1}-\psi_{i-2} \theta_{2}-\Phi$, 
$i=158, \psi_{i}=\psi_{i-1} \theta_{1}-\psi_{i-2} \theta_{2}+\phi \Phi, i=159$

$$
\begin{gathered}
(1-\phi B)\left(1-\Phi B^{79}\right)\left(Z_{t}-\mu\right)=\left(1-\theta_{1} B-\theta_{2} B^{2}\right) a_{t} \\
\hat{Z}_{t+1}=\left(1-\sum_{i=1}^{\infty} \psi_{i} \mu\right)+Z_{t} \sum_{i=1}^{\infty} B \psi_{i}
\end{gathered}
$$

Where $\psi_{0}=1, \psi_{1}=\phi-\theta_{1}, \psi_{2}=\psi_{1} \theta_{1}-\theta_{2}$, and $\psi_{i}=\psi_{i-1} \theta_{1}-\psi_{i-2} \theta_{2}$ for $i>2, i \neq 79,80, \psi_{i}=$ $\psi_{i-1} \theta_{1}-\psi_{i-2} \theta_{2}+\Phi, i=79, \psi_{i}=\psi_{i-1} \theta_{1}-\psi_{i-2} \theta_{2}-\phi \Phi, i=80$.

Similarly, OCPB predictions are calculated from Eq. (17) where the conditional probability of each state given five previous speed observations weighs the forecasts from corresponding states obtained from Eq. (13). Model intercepts are updated based on Eq. (10) also in OCPB. OCPBr and OCPBr* additionally contain RLS for filtering ARIMA model parameters.

$$
\hat{Z}_{t+1}=\sum_{k=1}^{4} P\left(X_{t}=k \mid Z_{-1 . .-5}\right) \hat{Z}_{t+1, k}
$$

\subsubsection{Parameter filtering}

In the proposed method, RLS is applied to change $\operatorname{ARIMA}(1,0,2)$ model parameters (i.e., $\mu_{i}, \phi_{i}$, $\theta_{1 i}$, and $\theta_{2 i}$ for all states $i=1,2,3,4,5$. Parameters are adopted from Guo and Williams (2010) and carefully revised based on forecasting performances. All parameters are the same for $\mathrm{OCPBr}$ and $\mathrm{OCPBr}$ * except initial state covariances which are used as $\mathbf{P}_{t}=(\mathbf{1})_{4 x 4}$ and $\mathbf{P}_{t}=(\mathbf{0 . 0 0 1})_{4 x 4}$ respectively. Note that the ARIMAs are already state-by-state fits, therefore, it is not intended to have high jumps in parameter values. RLS changes parameters based on recursive calculations and forecasting errors within $\min ( \pm 0.1,0.99)$ or $\max ( \pm 0.1,-0.99)$ intervals. Hence, parameter values change within $(-0.99,0.99)$. For an example, Fig. 7 gives filtering of 5-state $\mathrm{OCPBr}$ and $\mathrm{OCPBr}^{*}$ parameters on the additional loop data from F23AML2 for $\phi_{3}, \phi_{5}, \theta_{31}, \theta_{32}, \theta_{51}$, and $\theta_{52}$ which are parameters of state 3 and 5 ARIMA models respectively. As it can be deduced from the figures, ARIMA parameters are filtered only state-by-state (e.g., $\left.P\left(X_{t}=k \mid Z_{-1 . .-5}\right)>10^{-4}\right)$ otherwise kept the values from previous time period.

\subsubsection{Computational time}

Major computational time is demanded by HMM in the proposed model. The training of HMM is performed using Baum-Welch algorithm which is a version of Expectation-Maximization algorithm. At each iteration, Baum-Welch algorithm calculates two probabilities: forward $(\alpha)$ and backward $(\beta)$. For a continuous model with $N$ hidden states and an input string of length $k$, the complexities for the probabilities are: $O_{\alpha}\left(N^{2} k\right)$ and $O_{\beta}\left(N^{2} k\right)$. The forward and backward probabilities are multiplied to calculate $\gamma\left(O_{\gamma}\left(N^{3} k\right)\right)$ and $\xi\left(O_{\xi}\left(N^{3} k\right)\right)$. At each iteration, $\gamma$ and $\xi$ are used to update the model parameters $\left(O\left(N^{3} k^{2}\right)\right)$, which gives the overall complexity of the algorithm to be $O\left(N^{3} k^{2} T\right)$, where $T$ is 

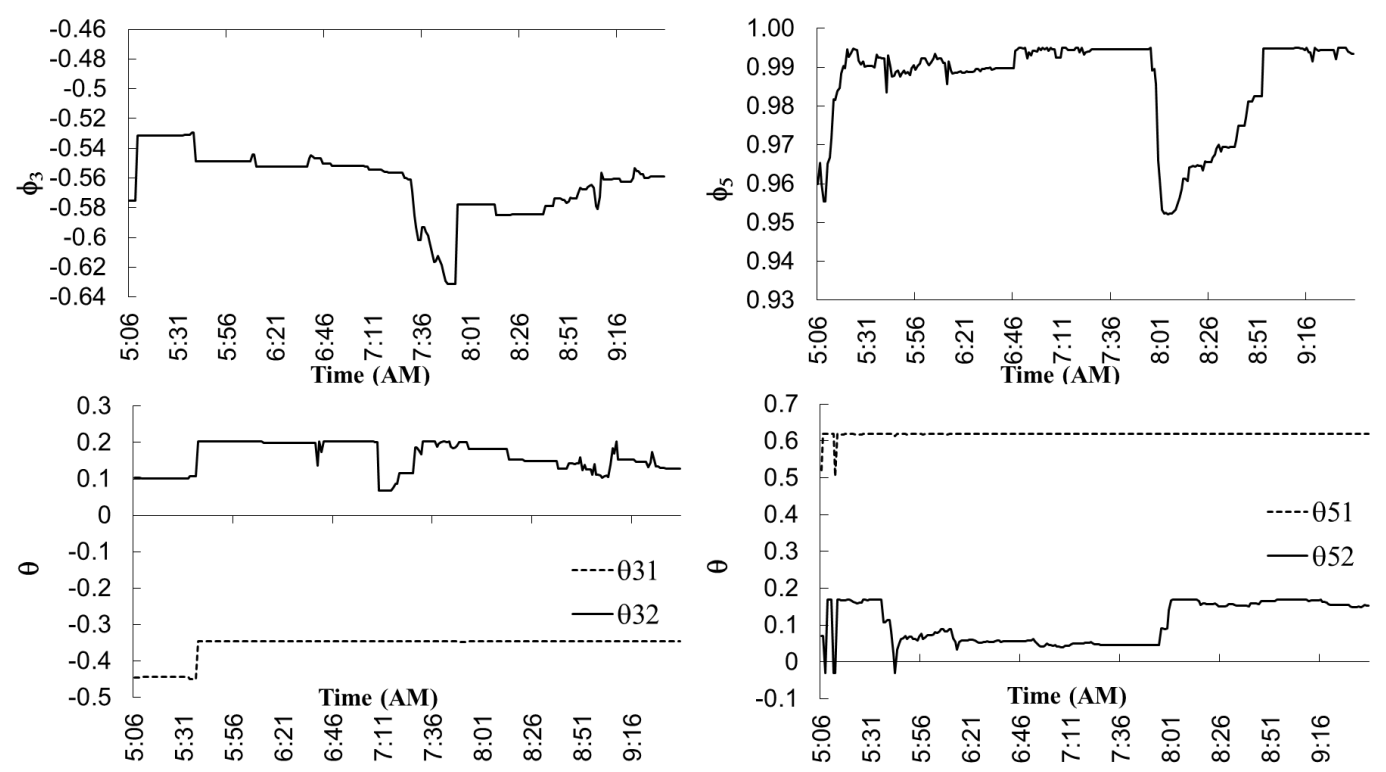

(a) $\mathrm{OCPBr}$
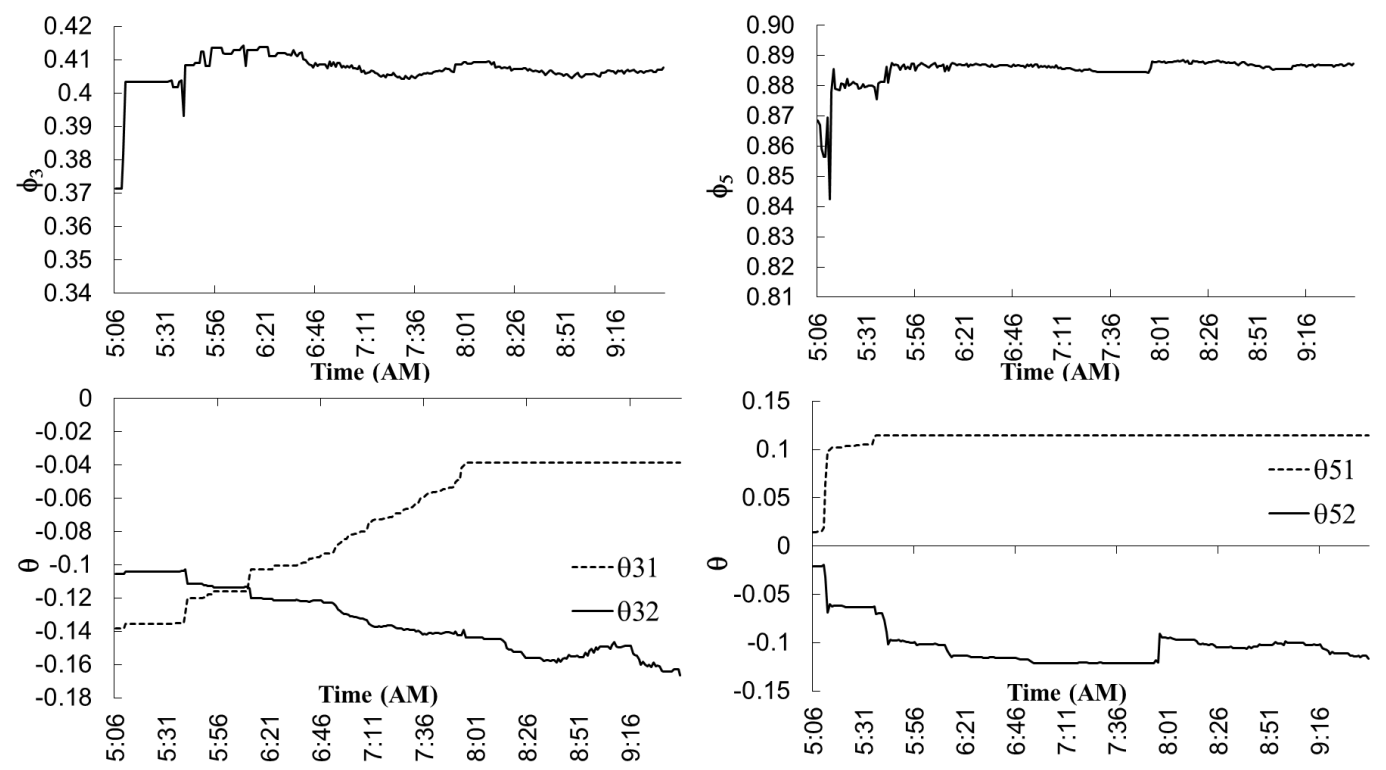

(b) $\mathrm{OCPBr}^{*}$

Fig. 7. (a) Examples RLS filtering of ARIMA model parameters with OCPBr on F23AML2. (b) Examples RLS filtering of ARIMA model parameters with OCPBr* on F23AML2.

the number of iterations. The training is performed on a PC with 8GB of memory, Pentium I3 QuadCore CPU and it takes under 20 seconds for all HMM configurations. EM algorithm in the developed method is input mixture of 10 fix historical data for distinguishable regimes and 3 new observations. Hence, convergence is very fast within a second to produce mean level prediction for a single time period. Fittings of times series models are also instantaneous when model is identified, e.g., ARIMA(1,0,2). HMM state prediction/probabilities, mean level estimation with EM, forecasting with time series models, and 
filtering all together about a second PC with 8GB of memory, Pentium I3 Quad-Core CPU for a single prediction period.

\subsection{Model testing and results}

The complete list of models used in the numerical experiments and their brief explanations are presented above in Table 1. The proposed method's without RLS and with versions with different number of states are referred as $\mathrm{OCPB} m$ and $\mathrm{OCPBr} m$ respectively. Selected 5-state models are denoted by $\mathrm{OCPBr}$ which is trained on the loop data and retrained $\mathrm{OCPBr}$ on INRIX data is referred as $\mathrm{OCPBr}$ * model given in Eq. (17).

In numerical examples first, OCPB models without and with RLS (i.e., OCPB and OCPBr) versions including different number of states are investigated. Six models (i.e., with 4, 5, and 6 states) are used to generate 1-step forecasts for the 38 days of speed data with various loop numbers and freeway sections given in Table 3. On the basis of the three error measures reported (i.e., mean square error $(\mathrm{MSE})=\sum_{t=1}^{n} \frac{\left(Z_{t}-\hat{Z}_{t}\right)^{2}}{n}$, mean absolute error $(\mathrm{MAE})=\sum_{t=1}^{n} \frac{\left|Z_{t}-\hat{Z}_{t}\right|}{n}$, and (MAPE) $\left.=\sum_{t=1}^{n} \frac{\left|Z_{t}-\hat{Z}_{t}\right|}{n Z_{t}}\right)$, Table 11 presents the summary of 1-step sample mean and standard deviations of prediction errors from compared models for several days. Fig. 8a shows the impact of number of states through distributions of errors. Box plots summarize the data with five points of $1^{\text {st }} Q_{1}, 2^{\text {nd }} Q_{2}$ median, $3^{\text {rd }} Q_{3}$ quartiles, upper, and lower limits for the extreme values. Upper and lower limits are calculated by $Q_{1}-1.5 I Q R$ and $Q_{3}+1.5 I Q R$ (interquartile range-IQR $=Q_{3}-Q_{1}$ ) which are approximately $\mu \pm 2.7 \sigma$ intervals for prediction errors. These results as well as the discussions in subsection 3.1.1 are used to determine the best option for number of states. Clearly, from the table and the figure it can be seen that 5-state model with RLS (i.e., $\mathrm{OCPBr}$ ) gives the highest accuracy improvement over 4-state OCPB model on average $27 \%$ in MSE and \%14 in MAE. Therefore, in the rest of the numerical analysis, $\mathrm{OCPBr}$ and $\mathrm{OCPBr} *$ are 5-state models.

\subsubsection{Compared forecasting methods}

The proposed method is compared with linear and nonlinear models. Linear models include mean adapting EM-ARIMA Eqs. (10) and (13), simple $\operatorname{ARIMA}(1,0,2)$ Eq. (13), $\operatorname{ARIMA}(1,1,1)$ Eq. (14), seasonal time series models of $\operatorname{SARIMA}(1,0,2)(1,1,0)$ Eq. (15), $\operatorname{SARIMA}(1,0,2)(1,0,0)$ Eq. (16), and two naïve models of Random Walk (RW) Eq. (18) and simple Moving Average (MA) Eq. (19).

$$
\begin{gathered}
\hat{Z}_{t+1}=Z_{t} \\
\hat{Z}_{t+1}=\sum_{i=0}^{4} Z_{t-i} / 5
\end{gathered}
$$

Nonlinear models used are logistic smooth transition autoregressive model (LSTAR) Eq.(20), self-exciting threshold autoregressive model (SETAR) Eq.(21), neural network nonlinear autoregressive model (NNTS) 
Table 11. 1-step forecasting performance of OCPB models

\begin{tabular}{ll|cccc}
\hline & & \multicolumn{2}{c}{ Error Summary } & \multicolumn{2}{c}{$\%$ Improvement } \\
\hline \multirow{2}{*}{ Model } & Error & Average & S. Deviation & Average & S. Deviation \\
\hline \multirow{2}{*}{ OCPB4 } & MSE & 10.39 & 5.22 & - & - \\
& MAE & 2.21 & 0.62 & - & - \\
\hline \multirow{2}{*}{ OCPBr4 } & MSE & 9.00 & 4.22 & $8 \%$ & $24 \%$ \\
& MAE & 2.20 & 0.54 & $-1 \%$ & $14 \%$ \\
\hline \multirow{2}{*}{ OCPB5 } & MSE & 9.74 & 5.06 & $5 \%$ & $12 \%$ \\
& MAE & 2.12 & 0.61 & $3 \%$ & $6 \%$ \\
\hline \multirow{2}{*}{ OCPBr5 } & MSE & 7.00 & 3.58 & $27 \%$ & $15 \%$ \\
& MAE & 1.84 & 0.45 & $14 \%$ & $8 \%$ \\
\hline \multirow{2}{*}{ OCPB6 } & MSE & 9.99 & 5.55 & $4 \%$ & $18 \%$ \\
& MAE & 2.19 & 0.67 & $1 \%$ & $7 \%$ \\
\hline \multirow{2}{*}{ OCPBr6 } & MSE & 7.78 & 4.00 & $23 \%$ & $13 \%$ \\
& MAE & 1.97 & 0.53 & $10 \%$ & $7 \%$ \\
\hline
\end{tabular}

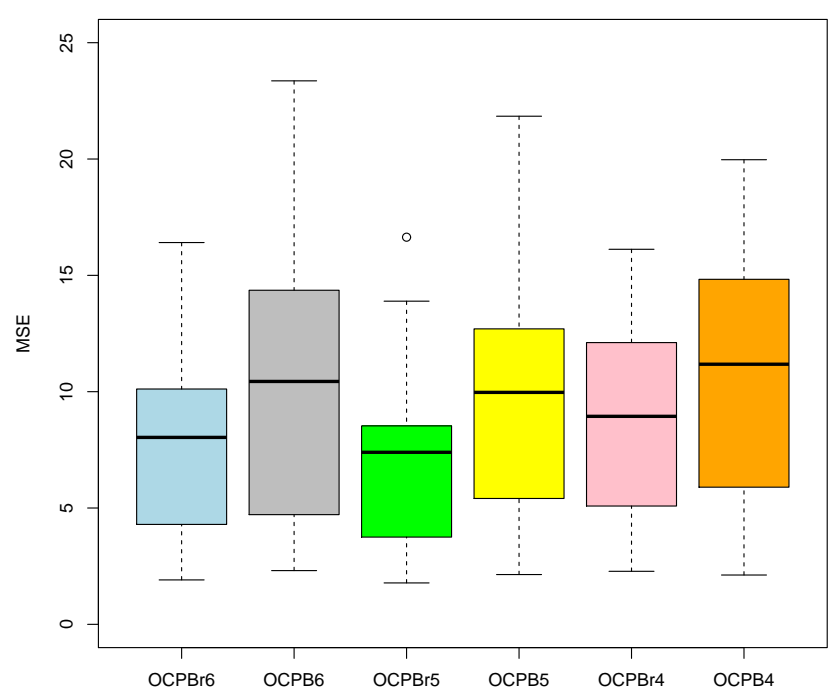

(a) Impact of number of states on the proposed method

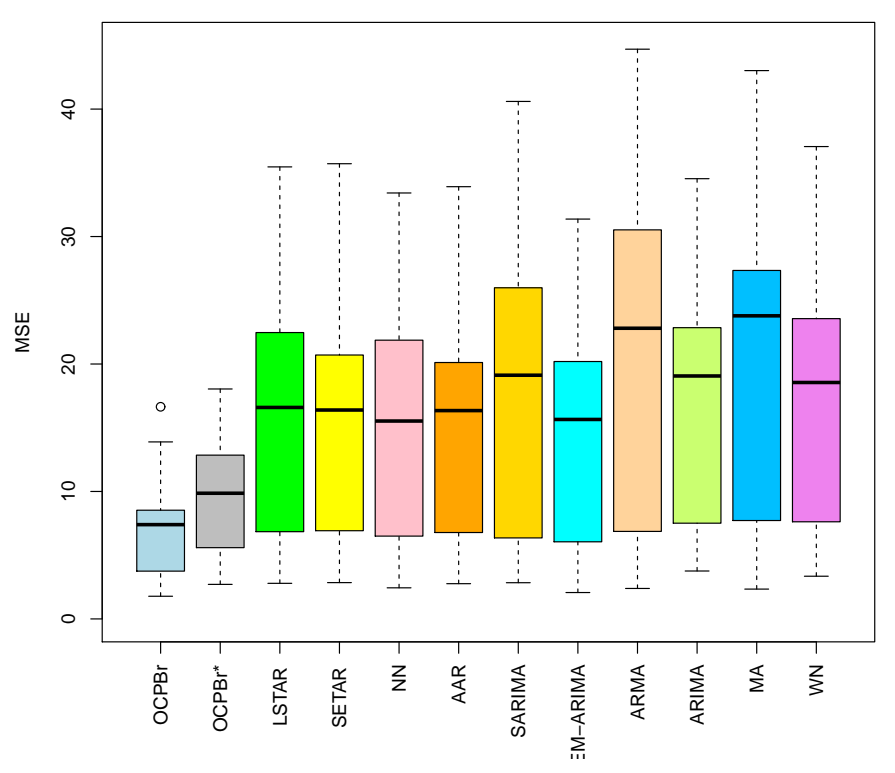

(b) All models compared for 1-step forecasts

Fig. 8. Box plots for 1-step forecasting errors on loop data

Eq.(22), and additive nonlinear autoregressive model (AAR) Eq.(23) which are adopted from Di Narzo et al. (2015). All of the models are fit to M3AM/PML4.

$$
Z_{t+1}=\left\{\begin{array}{l}
\left(\phi_{1}+\phi_{10} Z_{t}+\phi_{11} Z_{t-\delta}+\ldots+\phi_{1 L} Z_{t-(L-1) \delta}\right)\left(1-G\left(Z_{t}, \gamma, t h\right)\right)+ \\
\left(\phi_{2}+\phi_{20} Z_{t}+\phi_{21} Z_{t-\delta}+\ldots+\phi_{2 H} Z_{t-(H-1) \delta}\right) G\left(Z_{t}, \gamma, t h\right)+\epsilon_{t+1}
\end{array}\right.
$$


Where $G\left(Z_{t}, \gamma, t h\right)=\left[1+e^{-\gamma\left(Z_{t-1}-t h\right)}\right]^{-1}$ is logistics transition function.

$$
Z_{t+1}=\left\{\begin{array}{l}
\phi_{1}+\phi_{10} Z_{t}+\phi_{11} Z_{t-\delta}+\ldots+\phi_{1 L} Z_{t-(L-1) \delta}+\epsilon_{t+1}, X_{t} \leq t h \\
\phi_{2}+\phi_{20} Z_{t}+\phi_{21} Z_{t-\delta}+\ldots+\phi_{2 H} Z_{t-(H-1) \delta}+\epsilon_{t+1}, X_{t}>t h
\end{array}\right.
$$

Where $L=1$ to 5 and $H=1$ to 5 low and high regimes, $X_{t}$ is threshold function (the transition variable), $\delta$ delay of the transition variable, th is the threshold value.

$$
Z_{t+1}=\beta_{0}+\sum_{j=1}^{D} \beta_{i} g\left(\gamma_{0 j}+\sum_{i=1}^{m} \gamma_{i j}\left(Z_{t-(i-1) \delta}\right)\right)
$$

Eq. (22) shows the NNTS model where $m$ denotes embedding dimension, $D$ is hidden layer of the neural network, and $\beta_{i}, \gamma_{0 j}, \gamma_{i j}$ represent the weights.

$$
Z_{t+1}=\mu+\sum_{j=0}^{m-1} s_{j}\left(Z_{t-(j) \delta}\right)
$$

In Eq. 23, $s$ represents nonparametric univariate smoothing functions that depends on $Z_{t}$ s and $\delta$ is the delay parameter. Splines from Gaussian family are fitted in the form of $Z_{t+1} \sim \sum_{i=0}^{m-1} s\left(Z_{t}, . ., Z_{t-j}\right)$. Different $m$ values (i.e., $p+1$ for LSTAR and SETAR models) are fitted and based on their AIC/BIC, best models are selected. As shown in Fig. 9, for LSTAR $m=21$ and $m=3$ provide the lowest AIC and BIC values respectively. For SETAR, $m=15$ and $m=3$ for AIC and BIC min values. For AAR model, $m=3$ provides the min AIC and BIC values. NNTS model's $D$ hidden layer of the neural network is also optimized as shown in Fig. 9b within $D=3-20$ and $m=2-20$ values are used. Minimum AIC/BIC values for every $D$ are shown. According to $\mathrm{AIC} D=13, m=4$ is minimum where the best $\mathrm{BIC}$ is $D=4, m=4$. In addition, forecasting performances are checked and it was observed that both $m$ s for every model gave very close results. So, simpler models of LSTAR $m=3$, SETAR $m=3$, LINEAR $m=3$, NNETS $m=4, D=4$, AAR $m=3$, and NNTS $D=4$ and $m=4$ (i.e., fitted model contains 25 weights) are chosen to be compared against OCPBr models. Resulting fitted models are presented in Table 12. Nonlinear model fitting takes about 5 seconds for LSTAR, SETAR, and AAR. For NNTS it takes about 10 seconds. Grid optimization based on AIC and BIC for parameters of all nonlinear models for $m=3$-25 took about 15 minutes on a PC with 8GB of memory, Pentium I3 Quad-Core CPU.

For a quick comparison with linear and nonlinear models, Table 13 presents the overall average $\%$ improvements of $\mathrm{OCPBr}^{*}$ and $\mathrm{OCPBr}$ models in MSE and MAE over the compared models for 1-step predictions on loop dataset. Note that these are only overall averages. Since loop data contains gapped AM/PM periods, forecasts are actually done separately for AM and PM. In addition, Fig. 8b shows all of the methods compared through box plots. Clearly, $\mathrm{OCPBr}$ and $\mathrm{OCPBr}^{*}$ provide the lowest errors. From the Table 13, $\operatorname{ARIMA}(1,0,2), \operatorname{ARIMA}(1,1,1)$, and Seasonal ARIMA models are referred as ARMA, 


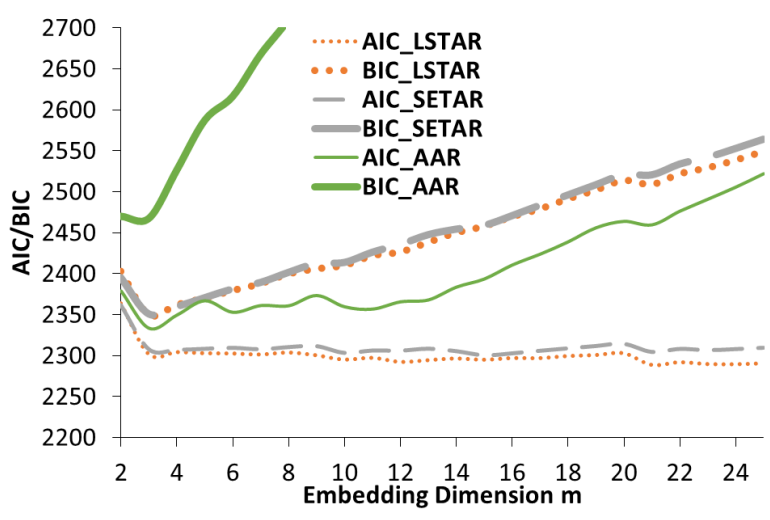

(a) Optimization of Nonlinear Time Series Fits

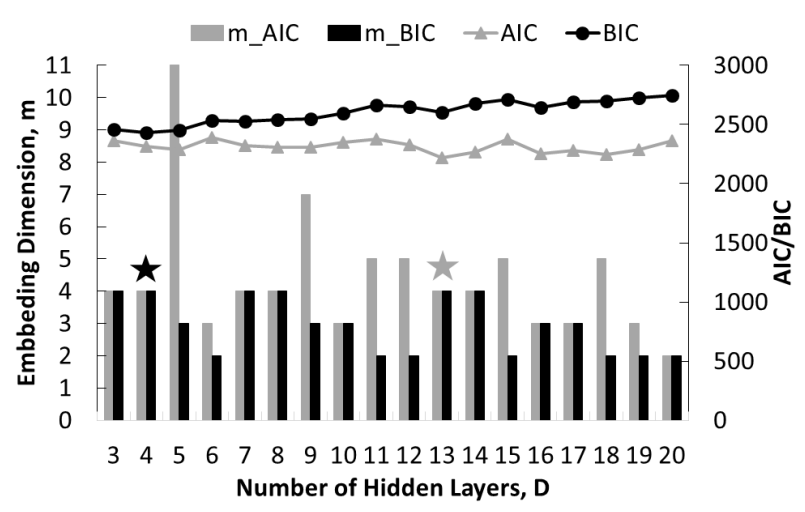

(b) Optimization of Neural Network Time Series Fits

Fig. 9. AIC and BIC values for Nonlinear Model Fitting

Table 12. Parameters for nonlinear models trained on M3AM/PML4

\begin{tabular}{ll|c}
\hline & Model & Parameters \\
\hline \multirow{2}{*}{$\mathrm{L}$} & $\phi_{1}=1.737 \phi_{10}=0.350 \phi_{11}=0.196 \phi_{12}=0.406$ \\
$\phi_{2}=-0.778 \phi_{20}=0.399 \phi_{21}=0.003 \phi_{22}=-0.373$ \\
$X_{t}=Z_{t}$, th $=35.22$
\end{tabular}

ARIMA, and SARIMA models respectively. From the table, proposed model shows on average $44 \%$ in MSE over EM-ARIMA, 55\%, over $\operatorname{ARIMA}(1,1,1), 56 \%$ over $\operatorname{ARIMA}(1,0,2), 54 \%$ over SARIMA, $57 \%$ over MA, and $55 \%$ over WN models. Over nonlinear models, predictions are at least $53 \%$ better in MSE and $26 \%$ in MAE. It is important to note that especially nonlinear time series models can have problem of getting stuck at a local optimum in fitting and they can perform better under some data types (Kuan (2002)). Hence, based on the sample data used in this study, following observations are presented for transferability, short-term, and long-term forecasting capabilities.

\subsubsection{Transferability}

In order to check the transferability, models are tested if they still perform well when the location is changed, i.e., moving from I-880 California Loop Data to Virginia INRIX Data. Furthermore, within loop dataset, the effect of refitting forecasting models at different loop sets is also tested. For this purpose first, the OCPBr model that is trained on loop dataset is used to predict speed for INRIX dataset. Then, the model is retrained on INRIX data (i.e., OCPBr*) and used for prediction. Furthermore, ARIMA and EM-ARIMA are also refit on INRIX data. Considering the observed differences in the datasets (see 
Table 13. 1-step forecasting performance of OCPBr5 over nonlinear and linear models

\begin{tabular}{|c|c|c|c|c|c|}
\hline \multirow[b]{2}{*}{ Model } & \multicolumn{3}{|c|}{ Error Summary } & \multicolumn{2}{|c|}{ \% Improvement } \\
\hline & Error & Average & S. Deviation & Average & S. Deviation \\
\hline \multirow{2}{*}{$\mathrm{OCPBr}$} & MSE & 7.00 & 3.58 & - & - \\
\hline & MAE & 1.84 & 0.45 & - & - \\
\hline \multirow{2}{*}{ OCPBr* } & MSE & 9.62 & 4.52 & $27 \%$ & $12 \%$ \\
\hline & MAE & 2.63 & 2.63 & $18 \%$ & $15 \%$ \\
\hline \multirow{2}{*}{ LSTAR } & MSE & 16.26 & 9.26 & $52 \%$ & $13 \%$ \\
\hline & MAE & 2.60 & 0.82 & $28 \%$ & $7 \%$ \\
\hline \multirow{2}{*}{ SETAR } & MSE & 15.89 & 8.98 & $51 \%$ & $13 \%$ \\
\hline & MAE & 2.54 & 0.79 & $27 \%$ & $8 \%$ \\
\hline \multirow{2}{*}{ NNTS } & MSE & 15.37 & 8.68 & $49 \%$ & $15 \%$ \\
\hline & MAE & 2.53 & 0.81 & $25 \%$ & $9 \%$ \\
\hline \multirow{2}{*}{ AAR } & MSE & 15.28 & 8.56 & $49 \%$ & $14 \%$ \\
\hline & MAE & 2.54 & 0.81 & $25 \%$ & $9 \%$ \\
\hline \multirow{2}{*}{ SARIMA } & MSE & 17.94 & 10.92 & $54 \%$ & $16 \%$ \\
\hline & MAE & 2.63 & 0.99 & $25 \%$ & $13 \%$ \\
\hline \multirow{2}{*}{ EM-ARIMA } & MSE & 14.42 & 8.59 & $44 \%$ & $17 \%$ \\
\hline & MAE & 2.43 & 0.79 & $22 \%$ & $10 \%$ \\
\hline \multirow{2}{*}{ ARMA } & MSE & 20.73 & 13.07 & $56 \%$ & $21 \%$ \\
\hline & MAE & 2.83 & 1.09 & $30 \%$ & $15 \%$ \\
\hline \multirow{2}{*}{ ARIMA } & MSE & 17.14 & 9.20 & $55 \%$ & $13 \%$ \\
\hline & MAE & 2.73 & 0.78 & $31 \%$ & $9 \%$ \\
\hline \multirow{2}{*}{ MA } & MSE & 20.51 & 12.45 & $57 \%$ & $19 \%$ \\
\hline & MAE & 2.77 & 0.97 & $30 \%$ & $12 \%$ \\
\hline \multirow{2}{*}{ WN } & MSE & 16.89 & 9.45 & $55 \%$ & $11 \%$ \\
\hline & MAE & 2.71 & 0.84 & $30 \%$ & $8 \%$ \\
\hline
\end{tabular}

section 2.1), proposed model shows acceptable performance. For INRIX data, predictions overall reveal low errors. Even though observed differences are noted for INRIX data, they are certainly useful to test the proposed model's transferability to other locations and data types.

Table 14 shows MSE, MAE, and \% improvements of the $\mathrm{OCPBr}^{*}$ model over $\mathrm{OCPBr}$, retrained ARIMA and EM-ARIMA models on INRIX data for 1-step to 45-step forecasts. Likewise, Table 15 illustrates MSE, MAE, and \% improvements of the OCPBr model over OCPBr*, loop-by-loop trained ARIMA* and EM-ARIMA* models for 1-step to 45-step forecasts. Moreover, Fig. 10 presents box plots for 1-step prediction errors for both data. Clearly in Fig. 10a, OCPBr* gives the lowest errors. From Fig. 10b, OCPBr and OCPBr* demonstrate very close results. They are lowers than refit EM-ARIMA and ARIMA models. The compiled results in Table 14 indicate that 1-step speed forecasts using $\mathrm{OCPBr}^{*}$ provide about $25 \%$ better accuracy. For longer term though 5-step to 45-step forecasts, OCPBr yields improved error values. For any scenario, proposed model is more accurate than refitted EM-ARIMA and $\operatorname{ARIMA}(1,0,2)$. The same idea is also tested by performances of $\mathrm{OCPBr}^{*}$ and $\mathrm{OCPBr}$ on additional 
loop data (i.e., days and locations) which are given in Table 15. In this evaluation, EM-ARIMA and $\operatorname{ARIMA}(1,0,2)$ models are also fit on every loop location and used for forecasting on the days from corresponding loop locations (see Table 8). Performances are then compared to $\mathrm{OCPBr}$ and $\mathrm{OCPBr}^{*}$ models. Surely, OCPBr is expected to be better under this scenario as it is trained on the loop data. Regardless, OCPBr* is assessed for its performance on a different location. From the table, only 45-step long-term prediction results are worse than loop-by-loop trained ARIMA* and EM-ARIMA* models. Overall, OCPBr improvements stay always positive up to 10-step forecasts and negative differences are mainly at 45-step forecasts about $10 \%$ worse than ARIMA models in MSE. Simple models look reasonable for long-term forecasting purpose as adaptive methods do not contain a special tool for possible incident duration prediction or clustering method for the most similar past day. Although both under-perform compared to $\mathrm{OCPBr}$ models, loop-by-loop recalibration of parameters for ARIMA or EM-ARIMA are not necessarily improving the performance of such models.

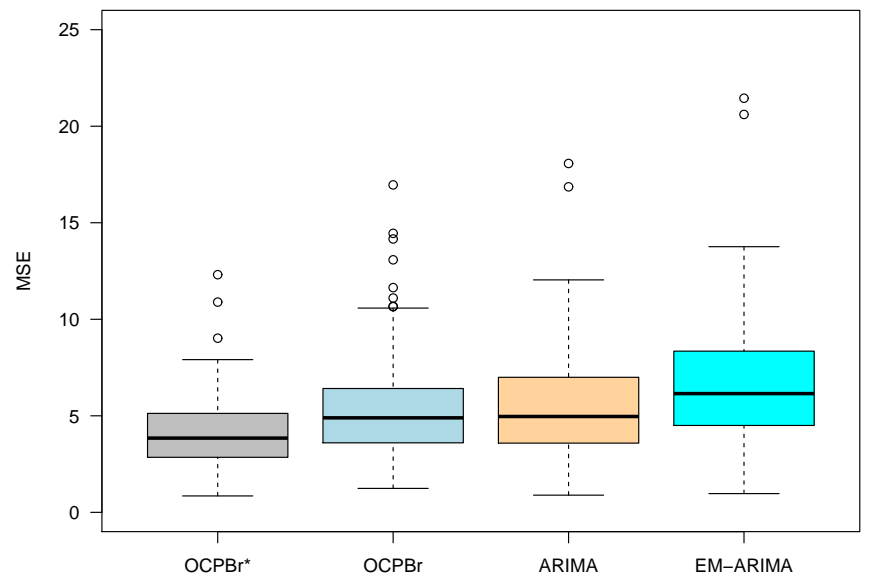

(a) MSEs of 1-step forecasts on INRIX data

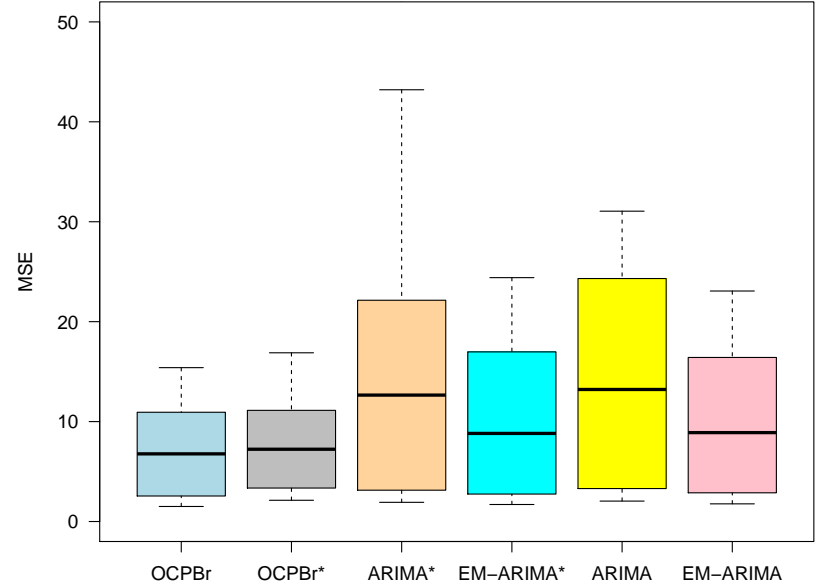

(b) MSEs of 1-step forecasts on Loop Data

Fig. 10. Box plots for 1-step prediction errors on INRIX and loop data

As far as transferability of $\mathrm{OCPBr}$ to different locations without retraining, the method looks to be promising candidate to employ for forecasting, if the dataset is subject to many changes. If the method originally trained at a dataset with clear changes like loop data, the results are better especially for shorter forecasting terms. Otherwise, the performance of the proposed model is very close to mean adapting EM-ARIMA model. 
Table 14. Summary of \% improvements for OCPBr* over OCPBr, ARIMA, and EM-ARIMA from INRIX data

\begin{tabular}{|c|c|c|c|c|c|c|c|c|c|c|c|c|c|}
\hline & \multicolumn{4}{|c|}{ OCPBr* } & \multicolumn{3}{|c|}{$\mathrm{OCPBr}$} & \multicolumn{3}{|c|}{ ARIMA } & \multicolumn{3}{|c|}{ EM-ARIMA } \\
\hline & & MSE & MAE & MAPE & MSE & MAE & MAPE & MSE & MAE & MAPE & MSE & MAE & MAPE \\
\hline \multirow{3}{*}{ 1-step } & AVG & 4.13 & 0.80 & 1.64 & 5.45 & 1.23 & 2.35 & 5.48 & 0.74 & 1.55 & 6.63 & 1.02 & 2.55 \\
\hline & SDEV & 1.95 & 0.20 & 0.56 & 2.83 & 0.26 & 0.63 & 2.92 & 0.19 & 0.54 & 3.53 & 0.32 & 1.65 \\
\hline & $\% \operatorname{Imp}$ & - & - & - & $23 \%$ & $35 \%$ & $31 \%$ & $34 \%$ & $19 \%$ & $25 \%$ & $22 \%$ & $-7 \%$ & $-6 \%$ \\
\hline \multirow{3}{*}{ 5-step } & AVG & 22.13 & 2.50 & 5.45 & 18.41 & 2.37 & 5.05 & 25.05 & 2.62 & 5.72 & 25.26 & 2.68 & 6.16 \\
\hline & SDEV & 11.71 & 0.61 & 2.16 & 9.56 & 0.56 & 1.89 & 12.98 & 0.65 & 2.26 & 13.14 & 0.68 & 2.76 \\
\hline & $\% \operatorname{Imp}$ & $17 \%$ & $5 \%$ & $7 \%$ & - & - & - & $27 \%$ & $10 \%$ & $12 \%$ & $27 \%$ & $12 \%$ & $18 \%$ \\
\hline \multirow{3}{*}{ 10-step } & AVG & 35.77 & 3.38 & 7.49 & 32.78 & 3.24 & 7.12 & 38.89 & 3.52 & 7.76 & 37.85 & 3.50 & 8.01 \\
\hline & SDEV & 20.31 & 0.89 & 3.25 & 18.85 & 0.86 & 3.04 & 21.71 & 0.92 & 3.30 & 21.16 & 0.92 & 3.75 \\
\hline & $\% \operatorname{Imp}$ & $8 \%$ & $4 \%$ & $5 \%$ & - & - & - & $16 \%$ & $8 \%$ & $8 \%$ & $13 \%$ & $7 \%$ & $11 \%$ \\
\hline \multirow{3}{*}{ 15-step } & AVG & 44.54 & 3.80 & 8.62 & 42.16 & 3.69 & 8.38 & 47.23 & 3.92 & 8.87 & 45.41 & 3.86 & 9.03 \\
\hline & SDEV & 27.52 & 1.06 & 4.06 & 26.17 & 1.04 & 3.93 & 28.56 & 1.08 & 4.14 & 27.44 & 1.07 & 4.56 \\
\hline & \% Imp & $5 \%$ & $3 \%$ & $3 \%$ & - & - & - & $11 \%$ & $6 \%$ & $6 \%$ & $7 \%$ & $5 \%$ & $7 \%$ \\
\hline \multirow{3}{*}{ 30-step } & AVG & 61.62 & 4.40 & 10.53 & 58.41 & 4.27 & 10.30 & 63.17 & 4.47 & 10.67 & 60.01 & 4.38 & 10.72 \\
\hline & SDEV & 45.57 & 1.41 & 6.15 & 44.75 & 1.45 & 6.11 & 46.00 & 1.41 & 6.19 & 43.49 & 1.39 & 6.51 \\
\hline & \% Imp & $5 \%$ & $3 \%$ & $2 \%$ & - & - & - & $8 \%$ & $5 \%$ & $3 \%$ & $3 \%$ & $3 \%$ & $4 \%$ \\
\hline \multirow{3}{*}{ 45-step } & AVG & 75.55 & 4.77 & 12.11 & 71.11 & 4.60 & 11.79 & 76.03 & 4.79 & 12.16 & 71.92 & 4.68 & 12.18 \\
\hline & SDEV & 60.85 & 1.71 & 8.11 & 58.85 & 1.72 & 8.02 & 60.83 & 1.71 & 8.14 & 57.22 & 1.67 & 8.43 \\
\hline & $\%$ Imp & $6 \%$ & $4 \%$ & $3 \%$ & - & - & - & $6 \%$ & $4 \%$ & $3 \%$ & $1 \%$ & $2 \%$ & $3 \%$ \\
\hline
\end{tabular}

Table 15. Summary of \% improvements for OCPBr over additional loop data

\begin{tabular}{|c|c|c|c|c|c|c|c|c|c|c|c|c|c|c|c|c|c|c|c|}
\hline & \multicolumn{4}{|c|}{$\mathrm{OCPBr}$} & \multicolumn{3}{|c|}{ OCPBr* } & \multicolumn{3}{|c|}{ ARIMA* } & \multicolumn{3}{|c|}{ EM-ARIMA* } & \multicolumn{3}{|c|}{ ARIMA } & \multicolumn{3}{|c|}{ EM-ARIMA } \\
\hline & & MSE & MAE & MAPE & MSE & MAE & MAPE & MSE & MAE & MAPE & MSE & MAE & MAPE & MSE & MAE & MAPE & MSE & MAE & MAPE \\
\hline \multirow{3}{*}{ 1-step } & AVG & 7.77 & 1.90 & 4.43 & 7.03 & 1.73 & 4.12 & 15.11 & 2.32 & 6.46 & 10.13 & 2.00 & 5.00 & 14.84 & 2.31 & 6.71 & 9.92 & 1.98 & 5.01 \\
\hline & SDEV & 4.54 & 0.51 & 2.52 & 4.72 & 0.54 & 2.56 & 13.28 & 1.08 & 5.72 & 7.56 & 0.75 & 3.40 & 14.40 & 1.16 & 6.85 & 7.26 & 0.73 & 3.55 \\
\hline & $\% \operatorname{Imp}$ & - & - & - & $23 \%$ & $12 \%$ & $11 \%$ & $38 \%$ & $20 \%$ & $24 \%$ & $22 \%$ & $11 \%$ & $13 \%$ & $39 \%$ & $19 \%$ & $24 \%$ & $23 \%$ & $11 \%$ & $13 \%$ \\
\hline \multirow{3}{*}{ 5-step } & AVG & 21.33 & 2.66 & 6.17 & 19.96 & 2.54 & 5.91 & 25.73 & 2.79 & 6.90 & 24.17 & 2.74 & 6.46 & 25.28 & 2.76 & 6.92 & 23.46 & 2.67 & 6.28 \\
\hline & SDEV & 19.96 & 1.19 & 4.32 & 19.00 & 1.19 & 4.21 & 25.38 & 1.44 & 5.43 & 23.72 & 1.36 & 4.79 & 25.17 & 1.44 & 5.72 & 23.37 & 1.32 & 4.64 \\
\hline & $\% \operatorname{Imp}$ & - & - & - & $9 \%$ & $5 \%$ & $5 \%$ & $10 \%$ & $6 \%$ & $8 \%$ & $9 \%$ & $5 \%$ & $6 \%$ & $10 \%$ & $5 \%$ & $8 \%$ & $6 \%$ & $3 \%$ & $3 \%$ \\
\hline \multirow{3}{*}{ 10-step } & AVG & 34.98 & 3.19 & 7.47 & 33.93 & 3.10 & 7.31 & 35.89 & 3.19 & 7.96 & 36.36 & 3.22 & 7.70 & 35.86 & 3.19 & 8.01 & 36.05 & 3.17 & 7.56 \\
\hline & SDEV & 35.56 & 1.71 & 5.73 & 34.90 & 1.70 & 5.68 & 37.16 & 1.81 & 6.65 & 37.65 & 1.81 & 6.13 & 37.27 & 1.84 & 6.89 & 37.70 & 1.80 & 6.01 \\
\hline & \% Imp & - & - & - & $6 \%$ & $3 \%$ & $3 \%$ & $0 \%$ & $1 \%$ & $3 \%$ & $2 \%$ & $2 \%$ & $3 \%$ & $0 \%$ & $1 \%$ & $3 \%$ & $0 \%$ & $1 \%$ & $1 \%$ \\
\hline \multirow{3}{*}{ 15-step } & AVG & 47.13 & 3.61 & 8.61 & 46.21 & 3.54 & 8.50 & 44.63 & 3.55 & 8.87 & 46.86 & 3.61 & 8.70 & 44.95 & 3.54 & 8.96 & 46.91 & 3.57 & 8.62 \\
\hline & SDEV & 49.71 & 2.16 & 6.98 & 49.11 & 2.16 & 7.00 & 47.99 & 2.20 & 7.74 & 50.29 & 2.22 & 7.32 & 48.61 & 2.23 & 8.08 & 50.89 & 2.23 & 7.29 \\
\hline & \% Imp & - & - & - & $5 \%$ & $3 \%$ & $3 \%$ & $-4 \%$ & $0 \%$ & $1 \%$ & $1 \%$ & $2 \%$ & $2 \%$ & $-3 \%$ & $-1 \%$ & $1 \%$ & $-2 \%$ & $0 \%$ & $0 \%$ \\
\hline \multirow{3}{*}{ 30-step } & AVG & 79.05 & 4.69 & 11.62 & 77.51 & 4.58 & 11.46 & 70.82 & 4.47 & 11.54 & 76.86 & 4.62 & 11.59 & 71.51 & 4.48 & 11.67 & 77.34 & 4.59 & 11.52 \\
\hline & SDEV & 93.61 & 3.39 & 11.15 & 92.54 & 3.39 & 11.17 & 86.18 & 3.30 & 11.56 & 93.43 & 3.43 & 11.37 & 86.66 & 3.33 & 11.88 & 94.03 & 3.43 & 11.31 \\
\hline & \% Imp & - & - & - & $7 \%$ & $4 \%$ & $4 \%$ & $-7 \%$ & $-2 \%$ & $-1 \%$ & $-1 \%$ & $1 \%$ & $1 \%$ & $-5 \%$ & $-2 \%$ & $0 \%$ & $-2 \%$ & $0 \%$ & $0 \%$ \\
\hline \multirow{3}{*}{ 45-step } & AVG & 108.36 & 5.72 & 14.20 & 106.31 & 5.55 & 13.92 & 93.97 & 5.29 & 13.70 & 103.68 & 5.51 & 13.90 & 95.17 & 5.32 & 13.89 & 104.70 & 5.52 & 13.91 \\
\hline & SDEV & 133.26 & 4.45 & 13.98 & 134.23 & 4.52 & 14.18 & 120.37 & 4.29 & 14.10 & 132.66 & 4.50 & 14.20 & 121.13 & 4.32 & 14.38 & 133.71 & 4.53 & 14.22 \\
\hline & $\% \operatorname{Imp}$ & - & - & - & $10 \%$ & $6 \%$ & $5 \%$ & $-11 \%$ & $-5 \%$ & $-3 \%$ & $-4 \%$ & $-1 \%$ & $-1 \%$ & $-9 \%$ & $-4 \%$ & $-2 \%$ & $-4 \%$ & $-1 \%$ & $-1 \%$ \\
\hline
\end{tabular}

\subsubsection{Short-term predictions}

Short-term prediction performance of the models are presented in this subsection. Conceptually, when there is an incident (major or minor), the forecasting errors for all models are larger compared to when there is no incident. Empirically comparing the data sets, this is more evident in loop dataset than INRIX dataset. ARIMA models consistently overestimate speeds when the mean level drops, while 
adaptive methods (i.e., OCPBr and EM-ARIMA) capture the changes in the mean level. Fig. 11 reveals this phenomenon as OCPBr follows the observed data closest. Other forecasting models become late as changes occur. From Figs. 8 and 10, MSE of ARIMA(1,0,2) reaches up to 43.21 on F23PML2 a day with two major shifts, whereas it is 2.87 for M1AML4 a day without a major change. MSE of ARIMA $(1,0,2)$ for INRIX data only reaches highest to 21.45 that is obtained on J22-5. For INRIX dataset, the average accuracy improvement over EM-ARIMA in MSE is 25\%. On J22-5, similar changes with long durations result up to $32 \%$ accuracy improvement over closest model EM-ARIMA. The lowest MSE for INRIX data is 0.97 from J20-0 with average MSE is about 6.63. Fig. 11 shows 1-step predictions on F23AML2 and J24-0. From the figure, on F23AML2 an incident causes a shift up to state 2 and generally within states 3, 4, and 5 (listed as major change in Table 3). The impact on the process is captured by OCPBr model with MSE of 13.30 versus the closest MSE of 15.53 (i.e., $14 \%$ difference) achieved by EM-ARIMA $(1,0,2)$ model. Similar process behavior on M15AML18 with a change to even lower speed (state 1) results in MSE difference of $37 \%$ over the next best model EM-ARIMA*. Overall, both OCPBr and OCPBr*
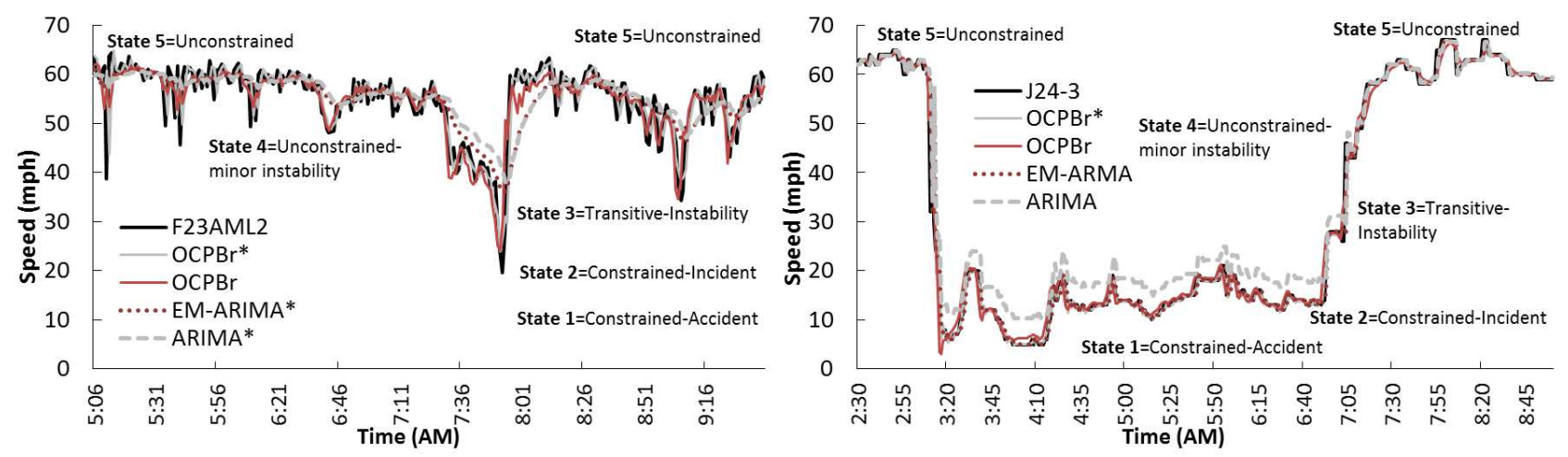

Fig. 11. 1-step predictions of aggregated speed of F23AML2 and INRIX J24-0

generate significantly better results than ARIMA and EM-ARIMA models even if they are refitted to the testing dataset. The OCPBr model outperforms nonadaptive models with average improvements reach up to $39 \%$ in MSE, 20\% in MAE and surpasses EM-ARIMA's performance with improvements reach up to $23 \%$ in MSE and $11 \%$ in MAE for 1-step predictions. From Tables 14 and 15, it can be observed that the improvements are mainly positive up to 15-step forecasts. There are only a few instances of negative improvements which are less than $5 \%$ on the days with no significant changes. The prediction accuracy gains reach up to $32 \%$ from EM-ARIMA, up to $43 \%$ from $\operatorname{ARIMA}(1,0,2)$ on INRIX data and $76 \%$ from $\operatorname{ARIMA}^{*}(1,0,2), 59 \%$ from EM-ARIMA*, 71\% from $\operatorname{ARIMA}(1,0,2)$, and $43 \%$ from EM-ARIMA on additional data. The difference observed between minor and major changes are only classified based on the impact reaching to state 1 (major change) or state 2 (minor change) and 3 (major instability). Certainly, multiple minor changes can also lead to higher improvements. For example, F24PML7 has 
multiple minor changes with short durations and M18PML18 has multiple minor changes with high variance and long duration impacts. On these two days, $\mathrm{OCPBr}$ model provides up to $62 \%$ improvement in MSE.

\subsubsection{Long-term predictions}

The models with a simple modification are tested for 5-45-step prediction results. Forecasts are calculated with current observations and historic average of five consecutive speed measurements from the same day of the past week given in Eq. (24). Similar idea is tested in Pan et al. (2012). Historical data and current observations are weighted in $\breve{Z}_{t+L}$ by $(1-\alpha)=0.05$ and $\alpha=0.95$ respectively. This is a design parameter which can be further investigated such as statistically the most similar day. Although, it is beyond the scope of this paper, few scenarios are tested in the experiments and several $\alpha$ values are checked manually. Moreover, the idea of term scope versus $\alpha$ is also tested. In sum, value of $\alpha=0.95$ is found to be providing better mixing for prediction performances. Results in MSE, MAE, and MAPE from the two data sets are shown in Tables 14 and 15. The tables present the overall errors, standard deviations, and average \% improvements of OCPBr* and OCPBr models in MSE, MAE, and MAPE over the compared models for 1-step to 45-step predictions on INRIX and loop datasets respectively.

$$
\breve{Z}_{t+L}=\alpha \hat{Z}_{t+L}+(1-\alpha) \sum_{i=0}^{4} Z_{t+L-i} / 5
$$

From the results, OCPBr is able to match the performance of retrained models. On INRIX data, OCPBr gives better forecasting results than OCPBr*. Accuracy on the additional data is also promising up to 10-step forecasts. The benefit of using OCPBr models over EM-ARIMA and nonadaptive methods decreases especially after 10-step forecasts as proposed model does not contain any incident prediction mechanism. The OCPBr becomes also late to react to the changes for multiple step predictions. Percent improvements reduce to on average less than 5\% from EM-ARIMA. From nonadaptive ARIMA models, although the impact diminishes, it still stays higher than $10 \%$. Forecast encompassing form is used $Z_{t}-\hat{Z}_{1 t}=\gamma+\beta \hat{Z}_{2 t}+\epsilon_{t}$ where $\gamma$ and $\beta$ are model parameters and $\epsilon_{t}$ is $N\left(0, \sigma^{2}\right)$ white noise error. $\hat{Z}_{1 t}$ and $\hat{Z}_{2 t}$ are forecasts from proposed $\mathrm{OCPBr}$ and historical average models. Forecast encompassing tests the null hypothesis of $\beta=0$ that is whether historical mean is encompassed by OCPBr for different step forecasts (Newbold and Harvey (2008); Clements and Harvey (2010)). When hypothesis is rejected, there is evidence that both methods contributes to the forecasts and optimum $\alpha$ are approximated by $\sigma_{2}^{2} /\left(\sigma_{1}^{2}+\sigma_{2}^{2}\right)$ for $\sigma_{1}^{2}=\operatorname{Var}\left(Z_{t}-\hat{Z}_{1 t}\right)$ and $\sigma_{2}^{2}=\operatorname{Var}\left(Z_{t}-\hat{Z}_{2 t}\right)$ (Newbold and Harvey (2008)). Table 16 shows the results for two days from loop and INRIX data. It can be seen that the contribution of historical average is encompassed by OCPBr until 15-step forecasts. For 30 and 45-step forecasts, the contribution of historical average is significant. In addition, $\mathrm{OCPBr}$ is using state probabilities where simple models 
are using either updated mean or only past observation. They are better to use for 30 and 45-step forecasts. However, these are obtained from single day results and combination coefficient of $\alpha=0.95$ used in the numerical examples seems reasonable.

Table 16. Forecast encompassing M17PML5 and J20-4

\begin{tabular}{lcccccccc}
\hline Interval & $\gamma$ & Loop Data- $\beta$ & $\mathrm{p}$-val & $\alpha$ & $\gamma$ & Inrix Data- $\beta$ & $\mathrm{p}$-val & $\alpha$ \\
\hline 1-step & 57.21 & 0.002 & 0.709 & 0.99 & 59.58 & 0.002 & 0.231 & 0.99 \\
5-step & 59.07 & -0.007 & 0.439 & 0.99 & 59.86 & $7.981 \times 10^{-04}$ & 0.76 & 0.99 \\
10-step & 58.43 & -0.009 & 0.387 & 0.98 & 60.14 & -0.001 & 0.761 & 0.99 \\
15-step & 58.46 & -0.014 & 0.233 & 0.98 & 60.41 & -0.003 & 0.472 & 0.99 \\
30-step & 62.01 & -0.041 & 0.001 & 0.98 & 62.28 & -0.012 & 0.020 & 0.98 \\
45-step & 65.94 & -0.075 & $<0.001$ & 0.97 & 62.70 & -0.016 & 0.010 & 0.97 \\
\hline
\end{tabular}

\section{Conclusions}

This paper develops an adaptive traffic parameter prediction model and demonstrates its effectiveness with extensive numerical evaluations. The proposed technique is a machine learning based method that involves HMM, EM, and RLS algorithms. It is able to improve the prediction accuracy significantly. In numerical examples, the model trained on the loop data is on average $44 \%$ and $22 \%$ better than EMARIMA, and more than 50\% and 30\% more accurate than ARIMA and naïve models. It also provides $23 \%$ and $40 \%$ more accurate forecasts than EM-ARIMA and ARIMA even if they are trained on each loop. For INRIX data, the improvement is about $19 \%$ and $30 \%$ over EM-ARIMA and ARIMA models. Up to 15-step predictions the differences stay positive for loop data and up to 30-step the improvements are positive for INRIX data. Over nonlinear time series models, the proposed model is more accurate at least $53 \%$ in MSE and \%26 in MAE. A basic weighted historical average is also used in long-term predictions. After 30-step forecasts, there is no apparent advantage over ARIMA or EM-ARIMA. However, the model does not perform worse either. The study additionally addresses the optimum number of states. Based on the data used, five-state models give the best results and improve the accuracy on average $14 \%$ and $27 \%$ in MSE without and with recursive least squares filtering respectively. Re-training of HMM and re-estimation of ARIMA model parameters for different locations on the network are also studied under transferability. Accuracy is found to be better until 15-step predictions over nonadaptive and mean adaptive models even for the datasets from different locations. The model outperforms loop-by-loop fit ARIMAs and EM-ARIMAs. Proposed model can be used without re-trained especially for longer than 1-step forecasts. Recalibration interval of ARIMA model parameters for a specific location can be counted as partially answered by employing a filtering algorithm. 
As well as the advantages, static parameters of HMM makes the model limited for significantly different change patterns. Without retraining, adaptive HMM transition matrix would enhance the performance especially for different locations. Number of data points required to train the complete model is large as it requires adequate sample size for each regime. For future work, multiple variables can be observed to supply more information for the state probability calculations. In addition, the duration of the incidents can be incorporated into change point models for better detection capability.

\section{Acknowledgments}

This paper is based upon work supported by the National Science Foundation under Grant No. 1238705, NSF Grant No. 1436222, and TranLIVE UTC Project No.521704. Any opinions, findings, and conclusions or recommendations expressed in this paper are those of the authors and do not necessarily reflect the views of the funding agencies.

Aiolfi, M., Capistran, C., Timmermann, A., 2011. Forecast combinations. In: Clements, M. P., Hendry, D. F. (Eds.), The Oxford Handbook of Economic Forecasting. Oxford University Press, Ch. 12, pp. $355-391$.

Baum, L., Eagon, J., May 1967. An inequality with applications to statistical estimation for probabilistic functions of Markov processes and to a model for ecology. Bulletin of the American Mathematical Society $73(3)$.

Baum, L. E., Petrie, T., Soules, G., Weiss, N., 1970. A Maximization Technique Occurring in the Statistical Analysis of Probabilistic Functions of Markov Chains. The Annals of Mathematical Statistics 41 (1), 164-171.

Bhotto, M. Z. A., Antoniou, A., 2013. New improved recursive least-squares adaptive-filtering algorithms. IEEE Transactions on Circuits and Systems 60 (6), 1548-1558.

Brook, D., Evans, D. A., 1972. An approach to the probability distribution of CUSUM run length. Biometrika 59 (3), 539-549.

Carpenter, S. R., Brock, W. A., Cole, J. J., Pace, M. L., 2014. A new approach for rapid detection of nearby thresholds in ecosystem time series. Oikos 123 (3), 290-297.

Castle, J. L., Fawcett, N. W., Hendry, D. F., 2011. Forecasting breaks and forecasting during breaks. In: Clements, M. P., Hendry, D. F. (Eds.), The Oxford Handbook of Economic Forecasting. Oxford University Press, Ch. 10, pp. 271-315. 
Celeux, G., Durand, J. B., 2008. Selecting hidden markov model state number with cross-validated likelihood. Computational Statistics 23, 541-564.

Cetin, M., Comert, G., 2007. Short-term traffic flow prediction with regime switching models. Transportation Research Record 1965, 23-31.

Clements, M. P., Franses, P. H., Swanson, N. R., 2004. Forecasting economic and financial time-series with non-linear models. International Journal of Forecasting 20, 169-183.

Clements, M. P., Harvey, D. I., 2010. Forecast encompassing tests and probability forecasts. Journal of Applied Econometrics 25 (6), 1028-1062.

Clements, M. P., Hendry, D. F., 1996. Intercept correction and structural change. Journal of Applied Econometrics 11 (5), 475-494.

Clements, M. P., Hendry, D. F., 2011. Forecasting from misspecified models in the presence of unanticipated location shifts. In: Clements, M. P., Hendry, D. F. (Eds.), The Oxford Handbook of Economic Forecasting. Oxford University Press, Ch. 11, pp. 315-355.

Comert, G., Bezuglov, A., 2013. An online change point based model for traffic parameter prediction. IEEE Transactions on Intelligent Transportation Systems 14 (3), 1360-1369.

Cosslett, S. R., Lee, L.-F., 1985. Serial correlation in latent discrete variable models. Journal of Econometrics 27 (1), 79-97.

Dempster, A. P., Laird, N. M., Rubin, D. B., 1977. Maximum likelihood from incomplete data via the em algorithm. Journal of The Royal Statistical Society, Series B 39 (1), 1-38.

Di Narzo, A. F., Aznarte, J. L., Stigler, M., 2015. R package:tsdyn manual.

Dimitriou, L., Tsekeris, T., Stathopoulos, A., 2008. Adaptive hybrid fuzzy rule-based system approach for modeling and predicting urban traffic flow. Transportation Research Part C:Emerging Technologies $16,554-573$.

Elhenawy, M., Rakha, A., 2014. Congestion prediction using adaptive boosting machine learning classifiers. In: Transportation Research Board 93rd Annual Meeting. No. 14-0830.

Goldfeld, S. M., Quandt, R. E., 1973. A markov model for switching regressions. Journal of econometrics $1(1), 3-15$. 
Gu, W., Choi, J., Gu, M., Simon, H., Wu, K., 2013. Fast change point detection for electricity market analysis. In: Big Data, 2013 IEEE International Conference on. IEEE, pp. 50-57.

Guo, F., Krishnan, R., Polak, J., 2014a. A novel three-stage framework for short-term travel time prediction under 1 normal and abnormal traffic conditions 2. In: Transportation Research Board 93rd Annual Meeting. No. 14-1114.

Guo, J., Huang, W., Williams, B. M., 2014b. Adaptive kalman filter approach for stochastic short-term traffic flow rate prediction and uncertainty quantification. Transportation Research Part C:Emerging Technologies 43, 50-64.

Guo, J., Williams, B. M., 2010. Real-time short term traffic speed level forecasting and uncertainity quantification using layered kalman filters. Transportation Research Record 2175, 28-37.

Hamilton, J. D., 1989. A new approach to the economic analysis of nonstationary time series and the business cycle. Econometrica: Journal of the Econometric Society, 357-384.

Hamilton, J. D., 2008. Regime-Switching Models, 2nd Edition. Palgrave McMillan Ltd.

Hamilton, J. D., 2010. Calling recessions in real time. International Journal of Forecasting 27 (4), 1006 1026.

Hastie, T., Tibshirani, R., Friedman, J., 2009. The Elements Statistical Learning: Data Mining, Inference, and Prediction, 2nd Edition. Springer.

Kamarianakis, Kanas, Y. A., P., P., 2005. Modeling traffic volatility dynamics in an urban network. Transportation Research Record 1923, 18-27.

Kamarianakis, Y., Gao, H. O., Prastacos, P., 2010. Characterizing regimes in daily cycles of urban traffic using smooth-transition regressions. Transportation Research Part C:Emerging Technologies:Emerging Technologies 18, 821-840.

Karlaftis, M. G., Vlahogianni, E. I., 2009. Memory properties and fractional integration in transportation time-series. Transportation Research Part C:Emerging Technologies (17), 444-453.

Keshavarz, M., Huang, B., 2014. Bayesian and expectation maximization methods for multivariate change point detection. Computers \& Chemical Engineering 60, 339-353.

Kim, J., Mahmassani, H. S., 2014. A finite mixture model of vehicle-to-vehicle and day-to-day variability of traffic network travel times. Transportation Research Part C:Emerging Technologies 46, 83-97. 
Kim, S., Coifman, B., 2014. Comparing inrix speed data against concurrent loop detector stations over several months. Transportation Research Part C:Emerging Technologies 49, 59-72.

Kindzerske, M. D., Ni, D., 2007. Composite nearest neighbor nonparametric regression to improve traffic prediction. Transportation Research Record 1993, 30-35.

Kuan, C.-M., 2002. Lecture on the markov switching model. Institute of Economics Academia Sinica, $1-30$.

Liu, L., Wang, H., Qian, Z. S., Wei, H., January 2014. A hybrid short-term traffic speed forecasting model through empirical mode decomposition (emd) and autoregressive integrated moving average (arima). In: TRB 93rd Annual Meeting Compendium of Papers DVD. Vol. 1. Washington, D.C.

McLachlan, G., Peel, D., 2000. Finite Mixture Models. John Wiley and Sons, Inc.

Melnykov, V., Melnykov, I., 2012. Initializing the em algorithm in gaussian mixture models with an unknown number of components. Computational Statistics and Data Analysis 56, 1381-1395.

Min, W., Wynter, L., 2011. Real-time road traffic prediction with spatio-temporal correlations. Transportation Research Part C:Emerging Technologies 19 (4), 606-616.

Newbold, P., Harvey, D. I., 2008. Forecast combination and encompassing. In: Clements, M. P., Hendry, D. F. (Eds.), A Companion to Economic Forecasting. Blackwell Publishers, Ch. 12, pp. 268-283.

Noroozi, R., Hellinga, B., 2014. Real-time prediction of near-future traffic states on freeways using a markov model. Transportation Research Record 2421 (1), 115-124.

Paleologu, C., Benesty, J., Ciochina, S., 2008. A robust variable forgetting factor recursive least-squares algorithm for system identification. IEEE Signal Processing Letters 15, 597-600.

Pan, B., Demiryurek, U., Shahabi, C., 2012. Utilizing real-world transportation data for accurate traffic prediction. In: ICDM. pp. 595-604.

Park, B.-J., Zhang, Y., Lord, D., 2010. Bayesian mixture modeling approach to account for heterogeneity in speed data. Transportation Research Part B: Methodological 44 (5), 662-673.

Piger, J., 2009. Econometrics: models of regime changes. In: Complex Systems in Finance and Econometrics. Springer, pp. 190-202.

Potter, S., 1999. Nonlinear time series modelling: An introduction. Journal of Economic Surveys 13 (5), $505-528$. 
Qi, Y., 2010. Probabilistic models for short term traffic conditions prediction. Ph.D. thesis, Louisiana State University, Baton Rouge,LA.

Qi, Y., Ishak, S., 2014. A hidden markov model for short term prediction of traffic conditions on freeways. Transportation Research Part C:Emerging Technologies 43, 95-111.

Rabiner, L., Feb. 1989. A tutorial on HMM and selected applications in speech recognition. Proceedings of the IEEE 77 (2), 257-286.

Ruggieri, E., 2013. A bayesian approach to detecting change points in climatic records. International Journal of Climatology 33 (2), 520-528.

Smith, B. L., Demetsky, M. J., 1997. Traffic flow forecasting: Comparison of modeling approaches. Journal of Transportation Engineering 123 (4), 261-266.

Smith, B. L., Williams, B. M., Oswald, R. K., 2002. Comparison of parametric and nonparametric models for traffic flow forecasting. Transportation Research Part C:Emerging Technologies 10, 303-321.

Sun, L., Zhou, J., 2005. Developing multi-regime speed-density relationships using cluster analysis. Transportation Research Record 1934 (1), 64-71.

Tan, M., Wong, S. C., Xu, J. M., Guan, Z. R., Zhang, P., 2009. An aggregation approach to short-term traffic flow prediction. IEEE Transactions on Intelligent Transportation Systems 10 (1), 60-69.

Tong, H., 1990. Non-linear time series: a dynamical system approach. Oxford University Press.

Vlahogianni, E. I., Golias, J. C., Karlaftis, M. G., 2004. Short-term traffic forecasting: Overview of objectives and methods. Transport Reviews 24 (5), 533-557.

Vlahogianni, E. I., Karlaftis, M. G., 2011. Statistical Methods versus Neural Networks in Transportation Research: Differences, Similarities, and Some Insights. Transportation Research Part C:Emerging Technologies:Emerging Technologies 19, 387-399.

Vlahogianni, E. I., Karlaftis, M. G., Golias, J. C., 2014. Short-term traffic forecasting: Where we are and where were going. Transportation Research Part C:Emerging Technologies 43, 3-19.

Wang, J., Deng, W., Guo, Y., 2014. New bayesian combination method for short-term traffic flow forecasting. Transportation Research Part C:Emerging Technologies 43, 79-94.

Wang, J., He, Q. P., Edgar, T. F., 2009. State estimation in high-mix semiconductor manufacturing. Journal of Process Control 19 (3), 443-456. 
Wei, W. W., 2006. Time series analysis: Univariate and multivariate methods.

Williams, B. M., 2001. Multivariate vehicular traffic flow prediction: Evaluation of ARMAX modeling. Transportation Research Record (1776), 194-200.

Zeng, J., De Callafon, R., 2006. Recursive filter estimation for feedforward noise cancellation with acoustic coupling. Journal of sound and vibration 291 (3), 1061-1079.

Zhang, Y., Zhang, Y., Haghani, A., 2014. A hybrid short-term traffic flow forecasting method based on spectral analysis and statistical volatility model. Transportation Research Part C:Emerging Technologies $43,65-78$.

Zou, Y., 2013. A multivariate analysis of freeway speed and headway data. Ph.D. thesis. 